\title{
Most primary olfactory neurons have individually neutral effects on behavior
}

Tayfun Tumkaya ${ }^{1,4}$, Safwan Burhanudin ${ }^{1}$, Asghar Khalilnezhad', James Stewart ${ }^{1}$, Hyungwon Choi ${ }^{1{ }^{3}}$, Adam Claridge-Chang ${ }^{1,2,4,5}$

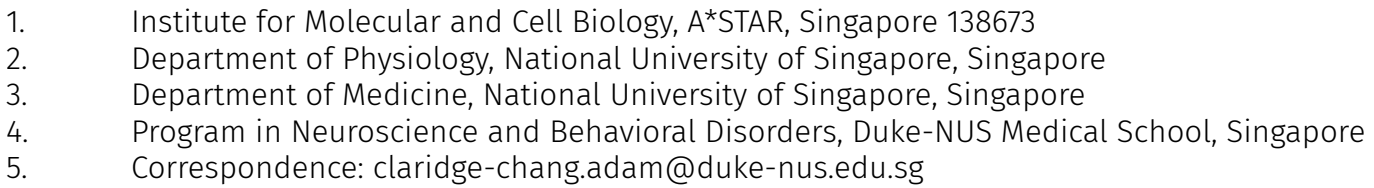

\section{Abstract}

Animals use olfactory receptors to navigate mates, food, and danger.

However, for complex olfactory systems, it is unknown what proportion of primary olfactory sensory neurons can individually drive avoidance or attraction. Similarly, the rules that govern behavioral responses to receptor combinations are unclear. We used optogenetic analysis in Drosophila to map the behavior elicited by olfactory-receptor neuron (ORN) classes: just one-fifth of ORN-types drove either avoidance or attraction. Although wind and hunger are closely linked to olfaction, neither had much effect on single-class responses. Several pooling rules have been invoked to explain how ORN types combine their behavioral influences; we activated two-way combinations and compared patterns of single- and double-ORN responses: these comparisons were inconsistent with simple pooling. We conclude that the majority of primary olfactory sensory neurons have neutral behavioral effects individually, but participate in broad, odor-elicited ensembles with potent behavioral effects arising from complex interactions. 


\section{Introduction}

Animals interact with their environment using motor functions that are guided by information that enters the brain from multiple sensory systems. These diverse sensory inputs are thought to interact with each other, with previously stored information, and with the internal physiological state of the animal to elicit a more or less appropriate behavioral response. Two central problems of neuroscientific research are (1) how individual sensations influence behavior, and (2) how multiple streams of sensory information are reconciled into meaningful behavior. The Drosophila olfactory system is an effective model to address these critical questions (Couto et al., 2005; Eisthen, 2002; Wang et al., 2003), facilitated by powerful genetic approaches, the ability to handle large sample sizes, and the numerical simplicity of Drosophila neural systems. Flies detect odors with their antennae and maxillary palps, which together contain $\sim 1300$ olfactory-receptor neurons (ORNs) (Lai et al., 2008). The odor-response profile of each adult ORN is determined by one of $\sim 45$ possible receptor types (Fishilevich and Vosshall, 2005; Gomez-Diaz et al., 2018). ORNs sharing the same receptor type converge on a glomerulus in the antennal lobe, where they synapse with local interneurons (LNs) and projection neurons (PNs) (Couto et al., 2005; Gao et al., 2000). Innervating throughout the antennal lobe and connecting multiple glomeruli, the LNs facilitate both excitatory and inhibitory interactions between glomeruli (Groschner and Miesenböck, 2019). This modified information is relayed by the PNs to higher brain centers, namely mushroom bodies and the lateral horn (Lai et al., 2008; Wang et al., 2014; Wong et al., 2002). The distinct nature of the ORN types allows us to consider each type as a single channel of information input. Mapping how ORNs steer behavior would inform a broader understanding of how sensory circuits influence behavioral output.

Odor-induced activity in ORNs can trigger approach and avoidance behaviors, collectively referred to as behavioral 
'valence' (Knaden et al., 2012). At least some ORN-driven behaviors appear to follow simple rules: a subset of receptors respond specifically to single odorants, and their ORNs individually drive innate valence (Ache and Young, 2005; Grabe and Sachse, 2018; Haddad et al., 2010; Haverkamp et al., 2018; Stensmyr et al., 2012; Suh et al., 2007). These acutely tuned, strongly valent ORN classes include neurons tuned to danger (e.g. toxic odorants) and pheromones. Given the direct relationship between such odors and valence, these ORN types and their associated downstream pathways have been termed 'labeled lines' (Grabe and Sachse, 2018; Hildebrand and Shepherd, 1997; Kurtovic et al., 2007). The existence of labeled lines proves that at least some olfactory behaviors follow simple ORN-activity rules.

Unlike labeled lines, many other olfactory receptors are broadly tuned to respond to many odorants, and most pure odorants evoke responses across many ORN classes (Hallem and Carlson, 2006). As most olfactory behavior relies on activity in ORN groups, there is the outstanding question of how individual channels contribute to an odor's overall valence. It is not known how much more complex multi-ORN valence is compared to the relative simplicity of labeled-line behavior. Earlier studies have looked at whether multi-glomerular olfactory valence could be explained by statistical models of ORN or PN activity patterns. Depending on the type of experiments, some found that valence could be explained by simple rules, e.g. weighted summation of larval ORN activity (Kreher et al., 2008). Other studies found no relationship between single-glomerulus properties and odor-evoked behavior, or invoked more complex models of antennal-lobe function (Badel et al., 2016; Knaden et al., 2012; Kuebler et al., 2012; Meyer and Galizia, 2012). Due to the many-many relationship of most odorants and ORNs, using natural odors to isolate single-ORN valence effects is challenging (Haddad et al., 2010; Knaden et al., 2012; Semmelhack and Wang, 2009; Thoma et al., 2014; Turner 
and Ray, 2009). One study overcame this challenge by activating single-ORN types optogenetically (Bell and Wilson, 2016). Using eight attractive ORN types the researchers found that two-way ORN valence combinations follow either summation or max-pooling; this supports the idea that olfactory valence arises from simple rules. Thus, both simple mechanisms (labeled lines, summation) and complex inter-channel interactions have been invoked to explain olfactory valence, but their relative importance remains controversial.

The present study had two primary aims: to map which single ORN types drive valence; and to examine the extent to which simple pooling rules govern ORN-valence combinations. To do so, we measured the valence coding of the primary olfactory system by optogenetically stimulating single ORN classes. In the wild, olfaction typically occurs in windy environments, and is influenced by hunger state, so we explored whether single-type-ORN valence is similarly contingent on these factors (Bell and Wilson, 2016; Sengupta, 2013). We activated pairs of ORN types to investigate how their combinations influence valence, and built statistical models of ORN interactions. All the results indicate that ORN-valence computations are complex. 


\section{Results}

An optogenetic behavior assay reports on sensory valence

We investigated which single classes of primary

chemosensory neurons can elicit preference behavior, and the capability of the present experimental system to replicate prior studies. Generating Drosophila lines that express the red-light-shifted channelrhodopsin CsChrimson (Chr) in different receptor neurons, we tested whether individual neuronal types drive attraction or avoidance. Flies were presented with a choice between light and dark environments in a wind- and light-induced self-administration response (WALISAR) apparatus (Figure $1 \mathrm{~A})$. We tested the validity of this approach with flies expressing the Chr channel under the control of the Gr66a-Gal4 driver line, which labels bitter-taste-sensing gustatory-receptor neurons (Moon et al., 2006) and has been previously reported to drive avoidance when artificially activated (Aso et al., 2014; Shao et al., 2017). In a sequence of 12 trials using three light intensities (14, 42, and $70 \mu \mathrm{W} / \mathrm{mm}^{2}$ ) and two airflow states (on/off), the experimental Gr66a>Chr flies displayed robust light avoidance (Figure 1B-F; Figure S1B-S1D; Figure S2). To benchmark the assay against a published method, we compared present data with prior results (Shao et al., 2017); the standardized effect size of Gr66a-neuron avoidance at the highest intensity (Cohen's $d=-2.70$ at 70 $\mu \mathrm{W} / \mathrm{mm}^{2}$ ) was almost as large as that of the previously reported valence response $(d=-3.63$; Figure S3A). This replication of aversive Gr66a-cell activation confirmed that this study's optogenetic-choice apparatus could be used to measure negative valence mediated by sensory neurons.

To benchmark attraction behavior, we used an Orco-Gal4 driver line that labels $\sim 70 \%$ of all ORNs (Larsson et al., 2004; Wang et al., 2003). Others have reported Orco-neuron valence results: one study showed no behavioral effect (Suh et al., 2007); another found attraction in the presence of a wind cue only (Bell and Wilson, 2016). In our experiments, at two higher light intensities (42 and 70 
A

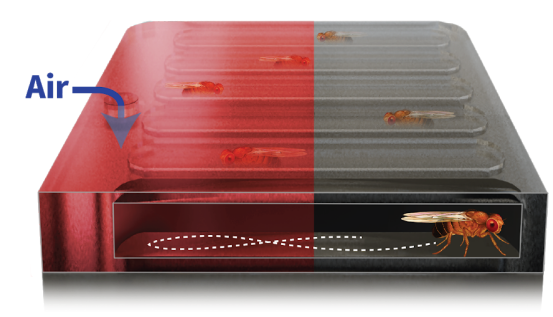

C

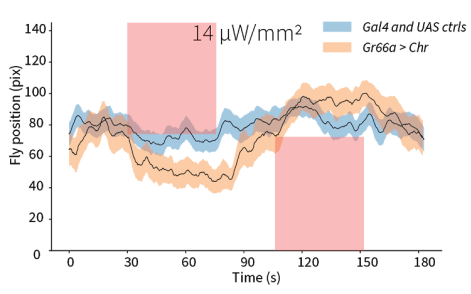

D

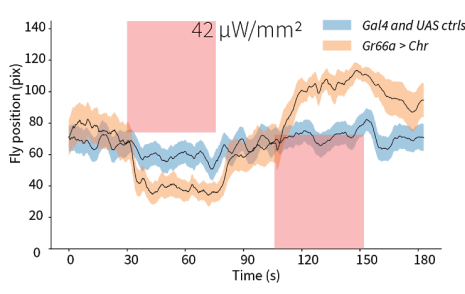

E

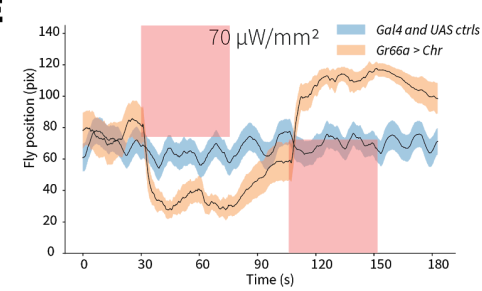

$\left.\mu \mathrm{W} / \mathrm{mm}^{2}\right)$, Orco>Chr flies exhibited pronounced attraction even in still air (Figure 2G; Figure S4). The valence was typically stronger than that reported in prior studies (e.g. $d$ $=0.56$ in still air in this study versus $d \leq 0.10$ ), establishing assay sensitivity for attraction (Figure S3B). Changing the temporal sequence of the 12 trials had negligible effects on Orco-neuron positive valence, suggesting that valence is not greatly susceptible to order bias, for example, due to habituation (Figure S4). Together with the Gr66a+ results, these data indicate that WALISAR is a valid, sensitive assay for measuring the valence of chemosensory circuits.

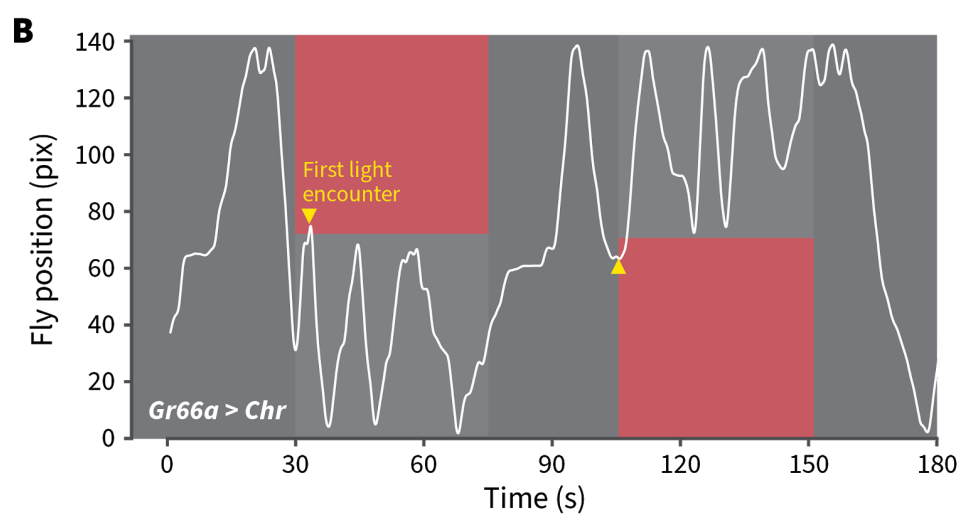

$\mathbf{F}$
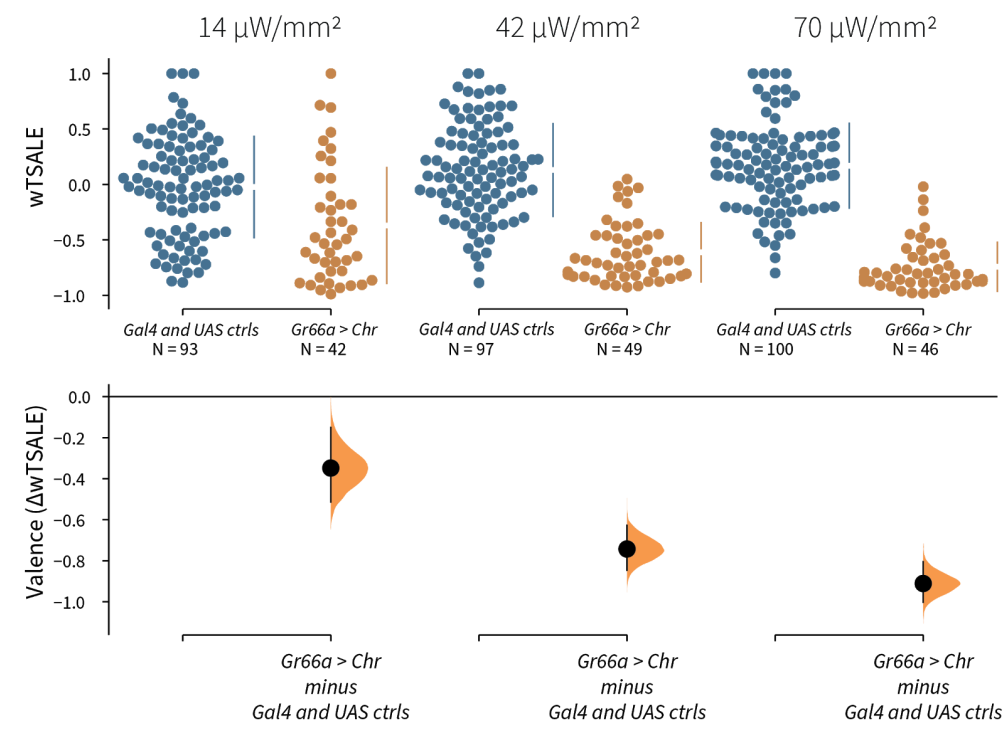

Figure 1. An optogenetic behavior assay reports on sensory valence

A. Individual flies were placed in WALISAR chambers and given a choice between no light or red-light illumination. 
B. A representative path of a fly displaying strong light avoidance. The white line represents the location of a Gr66a-Gal4 > UAS-CsChrimson fly in the chamber throughout an experiment. The light preference of each fly was calculated by how much time it spent in the illuminated zones after the initial encounter with light (yellow arrows).

C-E. Trace plots representing the mean location of controls ( $w^{1118}$; Gr66a-Gal4 and $w^{1118}$; UAS-CsChrimson), and test flies (Gr66a-Gal4 > UAS-CsChrimson) throughout an experiment at 14,42 , and $70 \mu \mathrm{W} / \mathrm{mm}^{2}$ light intensities. The blue and orange ribbons indicate $95 \% \mathrm{Cls}$ for the control and test flies, respectively.

F. An estimation plot presents the individual preference (upper axes) and valence (lower) of flies with activatable Gr66a+ neurons in the WALISAR assay. In the upper panel, each dot indicates a preference score (wTSALE) for an individual fly: ${ }^{1118}$; Gr66a-Gal4 and $w^{1118}$; UAS-CsChrimson flies are colored blue; and Gr66a-Gal4 > UAS-CsChrimson flies are in orange. The mean and $95 \% \mathrm{Cls}$ associated with each group are shown by the adjacent broken line. In the bottom panel, the black dots indicate the mean difference ( $\triangle$ WTSALE) between the relevant two groups: the valence effect size. The black whiskers span the $95 \%$ $\mathrm{Cls}$, and the orange curve represents the distribution of the mean difference.

\section{Only one-fifth of ORN types drive valence}

We aimed to estimate what proportion of single ORN types elicit valence when activated alone. Using available Gal4 lines, each driving expression in a single ORN type, we assessed the optogenetic valence of 46 receptor classes (Figure 2A). To separate the valence effects from noise, we analyzed data from $\sim 7,176$ flies with the Empirical-Bayes method (Figure 2B-C). The Empirical-Bayes analysis identified 10 valent classes: six ORNs elicited attraction and four elicited aversion (Figure 2A). The hits included six ORN classes with identified ligands. Four are considered labeled lines: Or56a, the receptor for the aversive odorant geosmin; Gr21a, the receptor for the aversive odorant carbon dioxide; Or67d, the receptor for the pheromone 11-cis-vaccenyl acetate; and Or47b, which senses the pheromone palmitoleic acid (Davis, 2007; van der Goes van Naters and Carlson, 2007; Jones et al., 2007; Kurtovic et al., 2007; Lin et al., 2016; Stensmyr et al., 2012; Suh et al., 2004). Additionally, Or83c mediates attraction to farnesol, an odorant produced by some ripe fruits (Ronderos et al., 2014), while Or42b mediates attraction to vinegar (Semmelhack and Wang, 2009). Given that this screen 
successfully recaptured six ORN types already known to be involved in ecologically-relevant valence functions, we consider that the screen was valid and sensitive.

Furthermore, the majority of the hits being ORNs with already-established valence implies that most ORNs are not singly valent.

To contextualize the screen's outcome, we conducted a literature review, tabulating prior and current results in ORN valence (Table S1). Although methodological diversity precludes a formal, quantitative meta-analysis (Borenstein et al., 2009; Tumkaya et al., 2018), it is clear that-for many ORNs-a consensus on valence is lacking. For example, the ORN screen showed that two additional, pheromone-responding ORNs (Or88a and Or65a) were not Empirical-Bayes hits (Chin et al., 2018; van der Goes van Naters and Carlson, 2007); however, no prior single-ORN data have shown these ORN types to individually drive valence (Table S1). It is possible that the behavioral effects of Or88a and Or65a depend on the presence of other cues or the activation of other receptors.

We only used male flies in the screen. Because odor responses in female flies might differ-especially for pheromonal receptors-we checked for possible sex differences in five receptor classes: Orco cells and four pheromone-responsive ORNs (Figure 2D-E). Although male flies exhibited strong responses to Orco-neuron activation, females showed no response. Surprisingly, this lack of response in females turned into a strong attraction when they were starved (Figure S5). Only one pheromone-receptor class showed sexual dimorphism: activation of Or47b neurons (sensors of an aphrodisiac pheromone) was attractive to males, while females were indifferent.

Together, these results indicate that in isolation, most ORN classes do not drive valence. The presence of six known valent ORN types in the 10 hits, and the predominance of neutral ORNs suggest that most olfactory channels 
influence behavior only when activated in concert as part of an odor-evoked ensemble. It should be noted, however, that this initial analysis was performed on flies measured in still air-an abnormal condition in the wild.

A

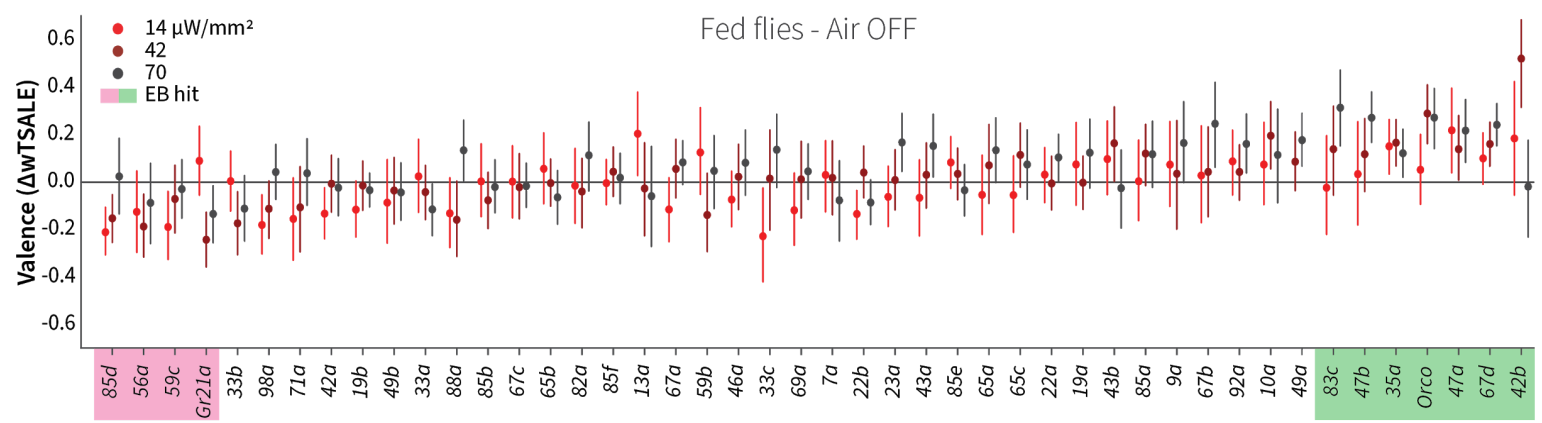

B

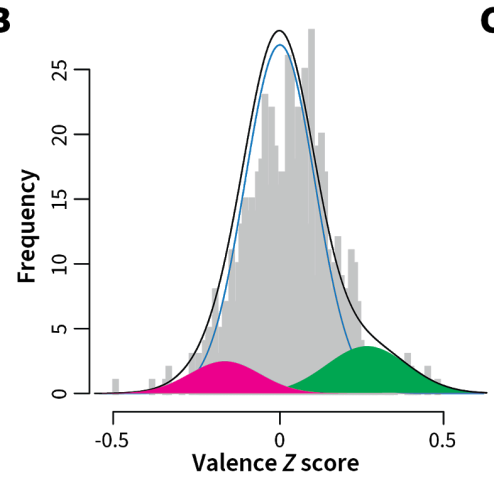

C

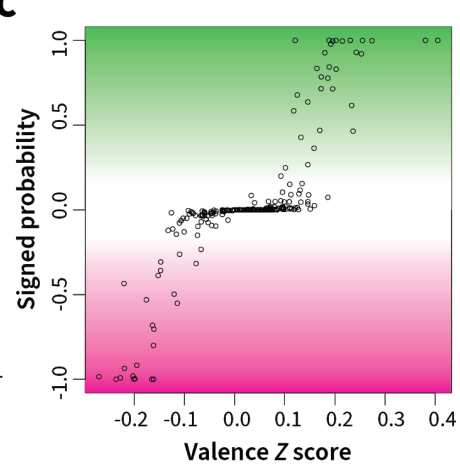

D

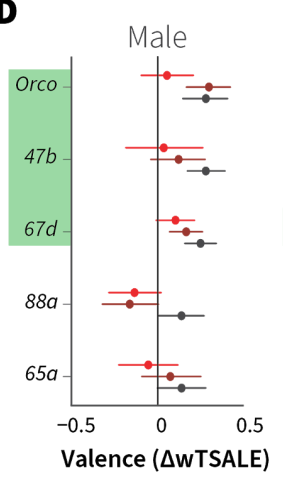

$\mathbf{E}$

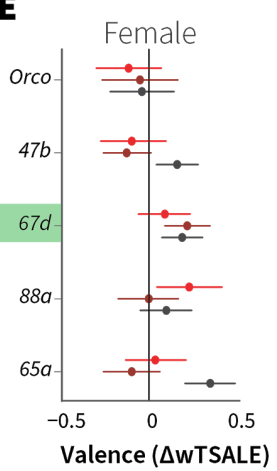

$\mathbf{F}$

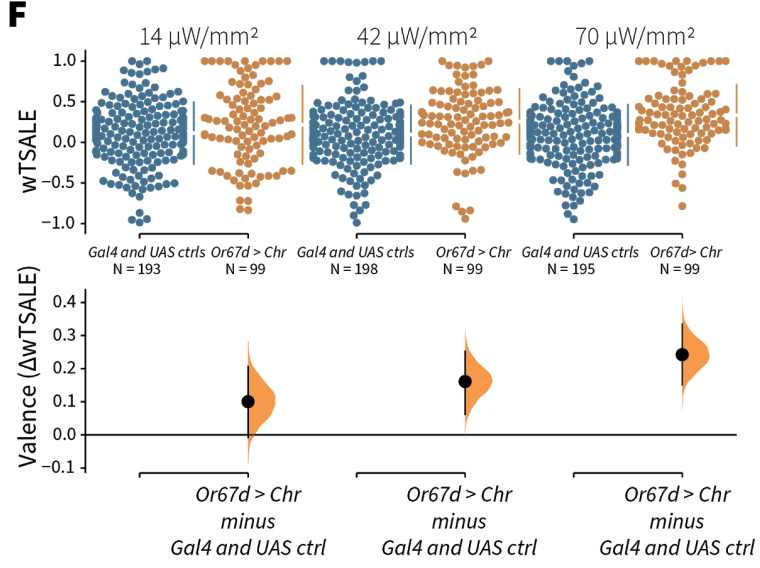

\section{G}
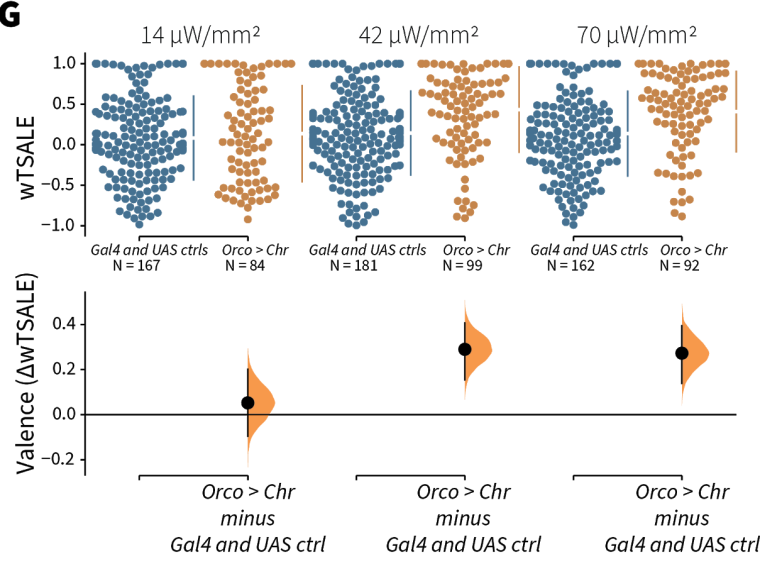

Figure 2. A small minority of ORN classes individually drive valence

A. An effect-size plot of the valence screen of 45 single-ORN types (and Orco neurons). Each dot represents a mean wTSALE difference of control $(\mathrm{N} \cong 104)$ and test $(\mathrm{N} \cong 52)$ flies, whisker indicate $95 \% \mathrm{Cls}$. The shades of red represent the three light intensities. Valent ORNs are shaded with magenta (aversion) or green (attraction).

B. The histogram (grey bars) of the median ORN $\triangle$ WTSALE ratios, and the mixture model that is fit to the data by Empirical-Bayes method. The blue line represents the null 
distribution, while the magenta and green curves represent ORNs with negative and positive valences, respectively. The black line represents the overall distribution of the effect sizes.

C. The signed posterior probability that each valence score is a true behavioural change, plotted against the respective effect size (median effect across the three light intensities). D-E. The olfactory valence of the Orco and four single-ORNs were tested in female flies (the male fly data is replotted from Panel A for comparison). The valence responses are represented as the mean difference ( $\triangle$ WTSALE) of control $(\mathrm{N} \cong 104)$ and test $(\mathrm{N} \cong 52)$ flies, along with $95 \% \mathrm{Cls}$. Color key is the same as above.

F-G. Estimation plots show the optogenetic preference (upper panel) and valence (lower panel) of flies with activatable Or67d and Orco neurons across three light intensities. The dots in the top panels represent single flies, while the broken lines indicate the mean and 95\% Cls. The differences between the pairs of test and control groups are displayed in the bottom panel, where the whiskers are $95 \% \mathrm{Cls}$ and the curves are the distributions of the mean difference.

\section{Wind does not amplify single-ORN valences}

It has been previously reported that wind is essential for the optogenetic valence of Orco neurons (Bell and Wilson, 2016). We thus aimed to test the hypothesis that wind amplifies ORN valence, possibly eliciting valence in some otherwise non-valent ORN classes. We tested this in the same flies by also measuring the valences of 46 ORN types in the presence of airflow (Figure 3A). From each fly, we used the paired wind-no-wind responses to calculate wind-specific effect sizes, $\Delta \Delta$, for each light intensity and each ORN type (Figure 3B). With a lone exception (wind rendered Or59b valence more aversive in the lowest light intensity only), an Empirical-Bayes model found that the wind effect sizes were indistinguishable from noise (Figure 3C-D). Contradicting our hypothesis, this result indicates that wind has essentially no impact on ORN-elicited behavior in walking flies. This result also generalizes the finding that, in either still or windy conditions, only a minority of ORN classes individually drive valence. 
A
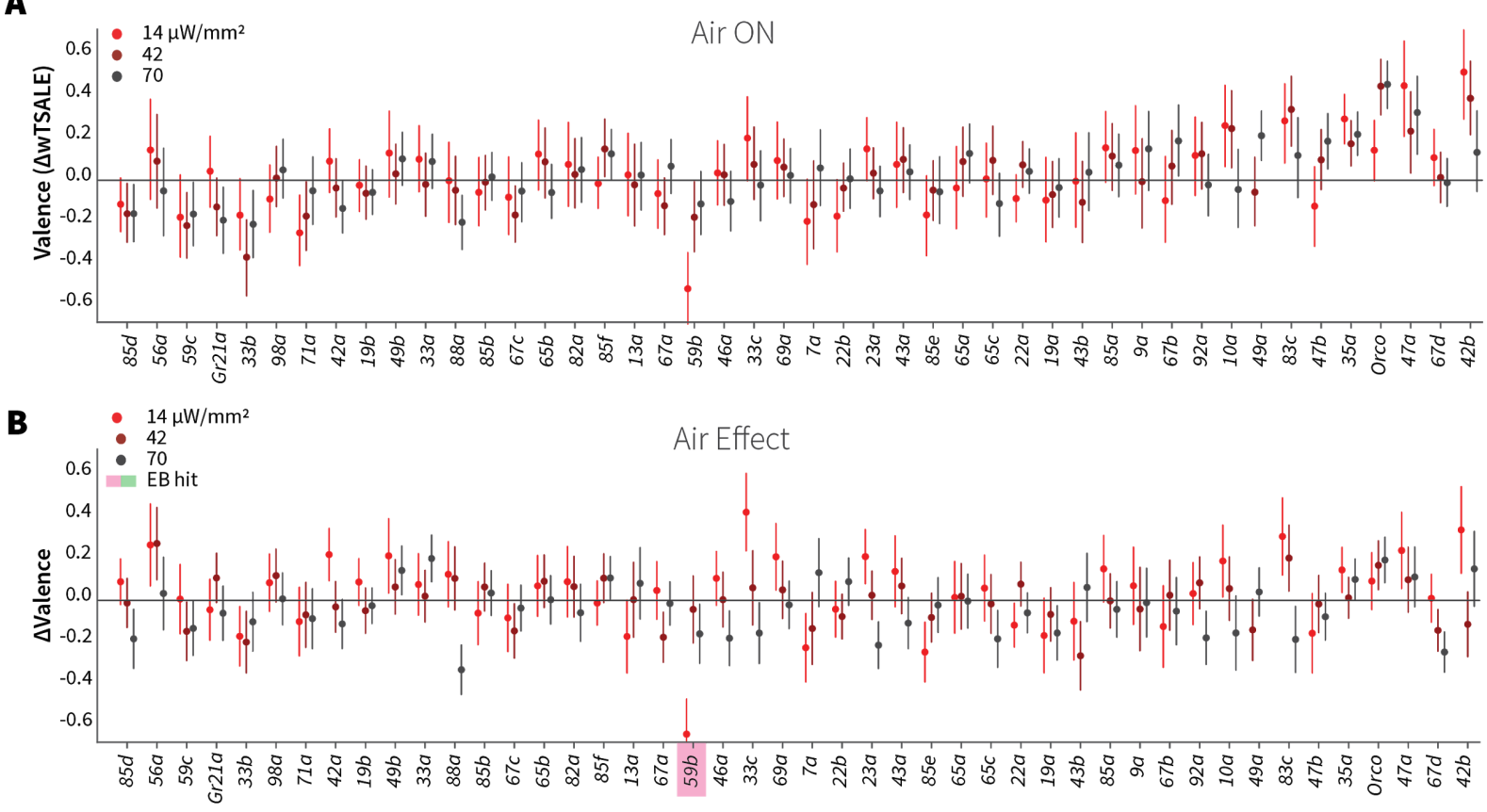

C

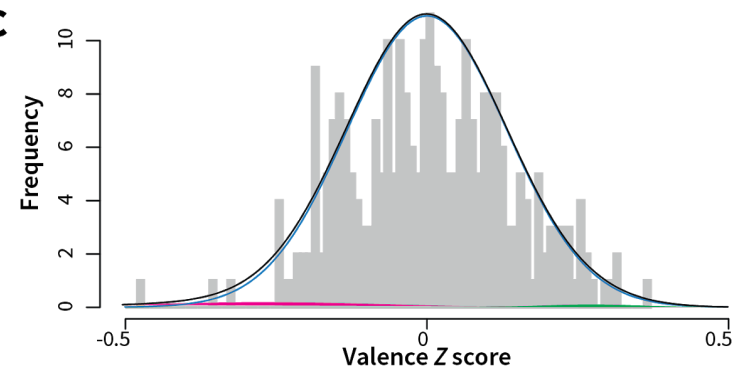

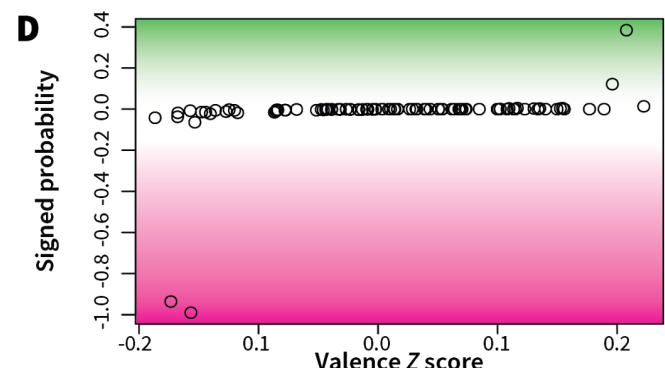

\section{Figure 3. Wind does not amplify single-ORN valences}

A. The results of ORN valence assays in the presence of airflow. The red dots represent the mean wTSALE differences of control $(\mathrm{N} \cong 104)$ and test $(\mathrm{N} \cong 52)$ flies with the $95 \% \mathrm{Cls}$.

B. The differences between the effect sizes of air-on and air-off experiments ( $\triangle \mathrm{WTSALE}$ ). The magenta shaded box indicates the sole Empirical-Bayes hit, Or59b, that showed an increase in aversion at the lowest light intensity only.

C. A statistical mixture model was fitted to the $\Delta \Delta w T S A L E$ scores. The grey bars are the response histogram. The magenta and green curves represent the effect sizes that differ from the null distribution (blue line). The black line represents the overall distribution of the $\triangle \triangle W T S A L E$ scores.

D. The signed posterior probabilities of the $\Delta \Delta w T S A L E$ scores being true behavioural changes versus their median effect sizes. The overall probability of true wind effects was nearly zero $[p(\Delta \Delta w T S A L E) \cong 0.0]$.

\section{Hunger has a limited effect on single-ORN valence}

Chemosensory behaviours-e.g. gustatory and olfactory responses-are influenced by an animal's internal energy 
state. Low internal energy, for example, can sensitize food odor-responsive ORNs and drive foraging (LeDue et al., 2016; Sengupta, 2013; Zhou et al., 2010). We hypothesized that starvation would thus increase the single-ORN valence behavior, especially for attractive ORN types. To test this hypothesis, we assayed $\sim 7,176$ starved flies for their optogenetic ORN valence (Figure 4A), and compared their behavior with that of the fed flies described above. Surprisingly, starvation did not enhance attraction. On the contrary, it reduced the responses triggered by three otherwise attractive ORNs: Or42b, Or47a, and Or83c (Figure 4B). Starvation also shifted the otherwise neutral valences of Or85f and Or49a, two receptors involved in sensing wasp odors (Ebrahim et al., 2015), into aversion (Figure 4B). Thus, hunger reduced the positive valence of a few pheromoneand food-sensing-ORNs, while slightly increasing the aversiveness of predator-sensing ORN classes. Overall, hunger does not have a broadly amplifying effect on single-ORN valence. 
A
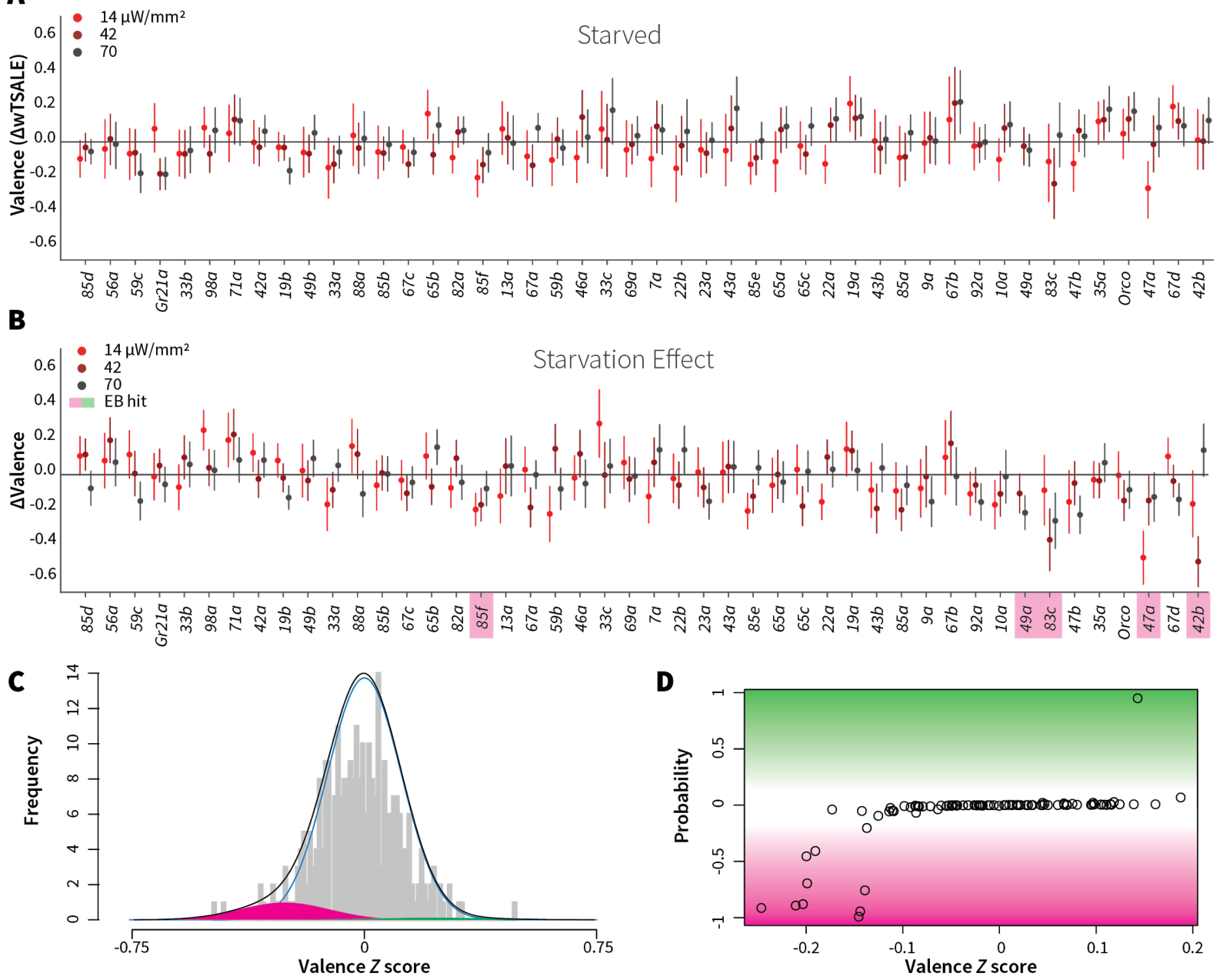

Figure 4. Starvation affects valence responses for five ORN classes

A. An ORN valence screen for starved animals. The red dots indicate the mean wTSALE differences between control $(\mathrm{N} \cong 104)$ and test $(\mathrm{N} \cong 52)$ flies.

B. The mean differences between fed and starved flies across ORNs. The magenta shading indicates the ORNs that are affected by starvation according to the Empirical-Bayes analysis.

C. A histogram of the median $\triangle \triangle W T S A L E$ ratio distribution. The blue line represents the null distribution, the magenta and green curves represent the distributions of negative and positive valences that are separated from the null distribution, and the black line represents the overall $\triangle \triangle W T S A L E$ distribution.

D. The signed posterior probability of the $\Delta \Delta$ wTSALE scores being true behavioral changes are plotted against their median ratios.

\section{ORN-valence combinations follow complex rules}

Because the ORN screens showed that most single ORN types do not drive valence individually, it would appear 
that activity across multiple sensory channels simultaneously is required to drive most

(non-labeled-line) olfactory behavior. One hypothesis of combinatorial odor valence holds that ORN-combination behaviors arise from simple two-way pooling rules: summation and max-pooling (both widely used in neural-net construction) (Bell and Wilson, 2016; Goodfellow et al., 2016).

To address this hypothesis, we asked how single-ORN valences are combined when two ORN classes are activated. We crossed eight ORN driver lines (three positive, two negative, and three neutral) so as to generate seven, two-way ORN combinations (henceforth "ORN-combos"). Compared to their constituent single ORNs, the ORN-combos elicited distinct valences (Figure $5 A, B, F)$. We modeled the combination effect sizes with three pooling functions: summation, max-pooling, and min-pooling (Figure 5C-4E). Strikingly, regression showed that none of the three functions could account for a large proportion of ORN-combo valence: summation, min-pooling, and max-pooling accounted for $0.2[95 \mathrm{Cl} 0.01$, 0.49 ], 0.18 [ $95 \mathrm{Cl} 0.0,0.5$ ], or 0.11 [95Cl $0.00,0.32$ ] of the variance in the observed ORN-combo results, respectively (Figure 5C-E). Furthermore, Bland-Altman method-comparison plots revealed wide limits of agreement (LoAs) between the observed and predicted ORN-combo valences by all three models: summation [SD1.96 -0.45, 0.28], max-pooling [SD1.96 -0.34, 0.13], min-pooling [SD1.96 -0.1, 0.35] (Figure S6). This analysis thus demonstrates that none of these three simple pooling rules are major predictors of how two-ORN odor valence emerges from single-ORN valence.

To generalize this analysis, we built multiple-linear-regression models of ORN-combo associations (Figure S7). In these models, the $\beta$ weights indicate the relative contribution strength of each of the two ORN classes. We drew scatterplots of the medians of $\beta$ values for the three light intensities (Figure 5K). If the 
combination valences arose from summation, we would expect the $\beta$ points to cluster along the diagonal (equal contribution); if combination valences followed max- or min-pooling, we would expect points clustering along the axes. However the $\beta$ points were dispersed, indicating a diversity of pooling rules. Moreover, as the light intensity increased, the $\beta$ points shifted further away from the diagonal (Figure 5K) and, in some cases, flipped dominance (Figure S8). For these ORN-type pairs, increasing intensity is associated with two phenomena: one of the two ORNs tends to become more dominant; and the combination rules are different at different activity levels. Thus the interactions between ORN pairs vary depending on receptor identity and stimulus intensity. 

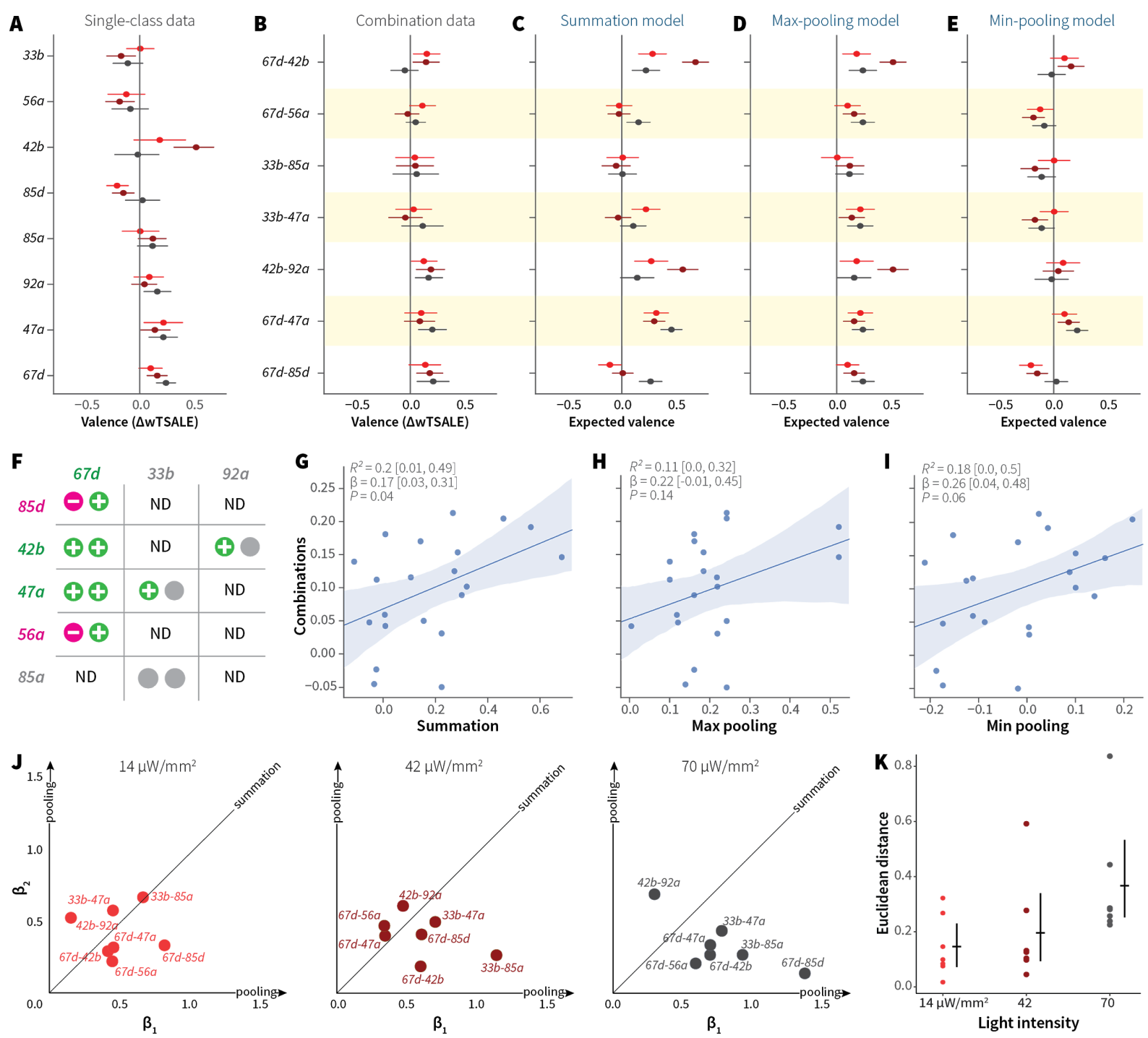

Figure 5. ORN-valence combinations follow complex rules

A. Valence responses of the single-ORN lines used to generate ORN-combos (replotted from Figure 1). The dots represent the mean valence between control $(\mathrm{N} \cong 104)$ and test $(\mathrm{N}$ $\cong 52$ ) flies ( $\triangle$ WTSALE with $95 \% \mathrm{Cls}$ ). The shades of red signify the three light intensities.

B. The valence responses produced by the ORN-combos in the WALISAR assay in three light intensities.

C-E. ORN-combo valences as predicted by the summation (C), max pooling (D) and min pooling (E) models.

F. Three positive (green), two negative (magenta), and three neutral (grey) ORNs were used to generate seven ORN two-way combinations.

G-I. Regression analyses of the experimental ORN-combo results and the predictions by the (G) summation, $R^{2}=0.2[95 \mathrm{Cl} 0.01,0.49], P=0.04 ;(\mathrm{H})$ max-pooling $R^{2}=0.11[95 \mathrm{Cl} 0.00$, 0.32 ], $P=0.14$; and (I) min-pooling $R^{2}=0.18$ [95Cl 0.0, 0.5], $P=0.06$ functions.

J. The scatter plots represent the influence of individual ORNs on the respective ORN-combo valence. The red, maroon, and black dots indicate ORN-combos at 14, 42, and 
$70 \mu \mathrm{W} / \mathrm{mm}^{2}$ light intensities, respectively. The horizontal $\left(\beta_{1}\right)$ and vertical $\left(\beta_{2}\right)$ axes show the median weights of ORN components in the resulting combination valence.

K. Euclidean distances of the ORN-combo $\beta$ points from the diagonal (summation) line in panel J. The average distance increases as the light stimulus intensifies: $0.14[95 \mathrm{Cl} 0.06$, 0.23 ], 0.20 [95Cl 0.06, 0.34], and 0.37 [ $95 \mathrm{Cl} 0.20,0.53$ ], respectively.

\section{Single ORN information predicts odor behavior poorly}

The variable interactions between pairs of ORN types support the idea that olfactory valence is dominated by complex dynamics in multiple layers of downstream circuits. So we anticipated that models using ORN activity would not be strongly predictive of odor valence. To explore this, we drew on data from two previous studies that used a panel of 110 odorants: one used the odor panel to make physiological recordings from 24 ORN types-of which 23 were tested for behavioral valence in this study (Hallem and Carlson, 2006); the other study measured behavioral valence for all 110 odors (Knaden et al., 2012). We adopted a partial-least-squares discriminant analysis (PLS-DA), to reduce the 23-dimensional feature space into fewer number of latent variables (LVs) with internal correlation (Figure S9A). Various models based on various numbers of LVs could partially predict odor preference. A multiple linear regression (MLR) model with eight LVs had the best performance, with an adjusted coefficient of determination $\left(\mathrm{R}_{\text {adj }}^{2}\right.$ ) of 0.23 . While the two-dimensional space defined by the first two LVs supports a partial separation of aversive and attractive odors (Figure S9B), the low $\mathrm{R}_{\text {adj }}^{2}$ score indicates that linear combinations of the available ORN-activity patterns are only weakly predictive of valence. The poor predictiveness of both the MLR model and a support-vector regression model were further confirmed by cross-validation (Table S2). For non-linear models, performance was limited by the relatively small sample size: two models with non-linear kernels (polynomial and radial basis function) had high error rates and learning curves indicating overfit (Table S2, Figure S10). Lastly, we asked whether the models could be improved with incorporation of the optogenetic valence data. 
Although valence-weighting improved the $\mathrm{R}^{2}$ adj of the MLR model from 0.23 to 0.33 , the predictive performances of the models were not improved (Table S3). This further supports the hypothesis that linear combinations of ORN activity can only account for a minority of odor preference.

\section{Discussion}

\section{Most ORN classes have no individual effect on valence}

This study's primary goal was to identify the proportion of ORNs that can drive valence behavior individually. The screen results indicate that 10/45 of single ORN-types have the ability to influence locomotor preference. Thus most ORN types are not individually valent. Of the ten valent ORN types, six have previously known roles in valence: two sensors each for pheromones, chemical-threat odorants, and food-aroma molecules. So the past two decades of Drosophila olfaction research have already identified a large proportion of strongly valent ORN types. The screen identified four novel valent ORNs (85d, 59c, 35a, 47a) with ligands and ecological roles yet to be determined. Around $60 \%$ of pure odorants are valent (Knaden et al., 2012), while at least three of the known valent ORN classes (those for geosmin, $\mathrm{CO}_{2}$, and $\mathrm{CVA}$ ) are specialized to bind specific ligands exclusively. This disconnect between a preponderance of valent odorants ( $60 \%$ of odorants) and the scarcity of broadly tuned, valent ORN types ( $\leq 15 \%)$ implies that most olfactory valence arises from combined activity in multiple ORN classes (Mathew et al., 2013; Parnas et al., 2013). This idea is also supported by the Orco activation result showing that broad activation across $\sim 70 \%$ of ORNs drives strong attraction.

\section{Wind and hunger effects on ORN valence are minor} Another goal of this study was to ask whether two contextual factors-wind and hunger-would increase olfactory valence. Prior studies observed that the Orco neurons are either not optogenetically valent or that airflow is essential for valence (Bell and Wilson, 2016; Suh et al., 2007). However, our experiments showed that 
olfactory neurons elicit valence without airflow, and that wind has little to no amplifying effect (Figure 1C, 2). Note also that even though Drosophila larvae behavioral tests are routinely performed in still air, they display both olfactory behavior and optogenetic ORN valence (Bellmann et al., 2010; Hernandez-Nunez et al., 2015; Mathew et al., 2013). Thus, the overall evidence indicates that Drosophila ORNs influence valence behavior even without wind. A second factor, hunger, has also been reported to increase olfactory attraction (Gelperin, 1971; Ko et al., 2015). However, in the single-ORN screen, starvation had modest effects on valence (Figure 4). If anything, valence was somewhat lower for several attractive ORNs (Figure 4B). There are at least two plausible explanations as to why starvation did not affect many single-ORN valences. First, the difference in olfactory responses between fed and starved animals might only be pronounced for weaker olfactory stimuli: one study showed that the ethyl acetate (EA) response difference between fed and starved flies declines as the EA concentration increases (Chakraborty, 2010). So optogenetic activation might be too strong to observe the starvation effect. Second, ORNs likely have less influence in isolation. Co-activated glomeruli modulate each other via lateral inhibition and excitation (Groschner and Miesenböck, 2019; Huang et al., 2010; Shang et al., 2007; Wilson, 2008). As most odors activate multiple ORN types, any hunger effect might require these lateral signals. One study found that five vinegar-responsive ORNs are modulated by hunger only when activated in concert, but not when they are activated in subsets (Root et al., 2011). In our results, a notable exception was the hunger switch of Orco valence in females (Figure S5). The absence of hunger amplification in single-ORN valence-and its presence in female Orco valence-also suggests that the potentiating effects of hunger on olfactory attraction might only operate on multi-ORN stimuli like Orco activation and natural odors. That neither wind nor hunger increased valence of single-ORNs verifies that the majority of single receptor types, on their own, do not convey valence 
information. Together, the single-ORN results support the idea that most odor-guided locomotion arises from the broad activation of ORNs simultaneously.

\section{How is ORN information combined?}

Many individual odorants bind multiple olfactory receptors, and most natural odors are complex blends that activate receptors broadly, such that odors typically activate multiple ORN classes. A number of groups have constructed statistical models of the relationship between initial layers of olfactory systems and their eventual valent locomotion (Badel et al., 2016; Bell and Wilson, 2016; Kreher et al., 2008; Kuebler et al., 2012; Kundu et al., 2016; Meyer and Galizia, 2012; Mohamed et al., 2019; Riffell et al., 2009; Thoma et al., 2014). The existence of labeled lines indicates that, at least for some odorants and their receptors, ORNs can have a deterministic effect on valence. In a model of Drosophila larval olfaction, the weighted summation of ORN activity in just five of 21 receptors could be used to predict odor valence (Kreher et al., 2008). In adults, an optogenetic study of ORNs reported that combination valences could be explained by unweighted summation and/or max-pooling (Bell and Wilson, 2016), further supporting a direct relationship. In contrast, the present analysis found little support for summation or pooling. Rather, the ORN-pair results point to a diversity of combination rules that vary over stimulus intensities, suggesting more complex ORN interactions.

\section{Technical differences between studies}

It is relevant to note that several of our conclusions on olfactory valence, notably the nature of ORN-valence combination, diverge from those made in an earlier study (Bell and Wilson, 2016). Along with sampling error, it is possible that these discrepancies could be attributed to differences in experimental design and analysis, 16 of which are summarized in Table S4. Here we discuss the different ways the optogenetic valence effect was controlled. As Chrimson was not available at the time, the earlier study used Channelrhodopsin-2 (ChR2), which 
requires intense blue light (up to $1500 \mu \mathrm{W} / \mathrm{mm}^{2}$ ) that adds significant heat and elicits strong responses in the fly visual system (Table S4). As these stimuli can profoundly alter behavior, especially during a lengthy illumination regime (64 min total), the researchers used genetically blind flies and an infrared laser for compensatory heat. While these technical measures were prudent, they were used largely in place of genetic controls: relative to the experimental animals $(\mathrm{N}=\sim 2512)$, the study used very few responder UAS controls $(\mathrm{N}=88)$ and zero driver Gal4 controls (Table S4). The earlier study also averaged technical replicates to represent behavioral variation, a procedure that under-reports variation (Bell, 2016). The present study dealt with these issues with two major differences. First, it was able to make use of the Chrimson channel, which requires lower light intensity (maximum 72 $\mu \mathrm{W} / \mathrm{mm}^{2}$ ) and a wavelength (red) to which the fly eye is less receptive. Second, all non-optogenetic effects were accounted for with balanced experimental, driver, and responder groups ( $\mathrm{N}=\sim 5148$ in each group). In our opinion, our approach of testing all three groups in sufficient sample sizes-and using them to calculate effect sizes-enables the exclusion of all confounding influences, including heat, visual effects, and genetic background.

\section{Do single-ORN-class properties govern valence?}

While larval valence has been predicted with a summation model, similar models for olfactory behavior in other systems have not been successful. Studies in various model animals have invoked complex computations to explain olfactory valence (Duchamp-Viret et al., 2003; Kuebler et al., 2012; Kundu et al., 2016; Meyer and Galizia, 2012; Riffell et al., 2009; Shen et al., 2013; Silbering and Galizia, 2007). In adult Drosophila, an analysis of the physiological and behavioral responses to 110 odors found that ORN activities and odor valence have no linear correlation (Knaden et al., 2012), suggesting that valence determination could arise from a downstream computation, for example in the antennal lobe, where incoming ORN activity patterns and outgoing 
projection-neuron activity patterns are dissimilar

(Groschner and Miesenböck, 2019). Indeed, the

physiological-activity patterns in projection neurons have been reported to be at least partially predictive of odor

valence (Badel et al., 2016; Knaden et al., 2012; Parnas et al., 2013), leading to the idea that the antennal lobe extracts valence features. To examine this, we modeled published ORN activity and valence data sets, finding that linear models of ORN activity data could account for a minor fraction of olfactory-behavior variance, and this was not substantially improved with single-class ORN valence information. All the observations in the present study point to a minimal role for simple pooling rules, and support the idea that odor valence is primarily governed by complex circuit dynamics. 


\section{Author contributions}

Conceptualization: TT, ACC; Methodology: TT, ACC, HC;

Experiments: TT, SBB, AK; Software: TT (Python, R), HC (R), JS (LabView); Data Analysis: TT; Hardware: TT, JS; Writing Original Draft: TT; Writing - Revision: TT, ACC; Visualization: TT; Supervision: ACC; Project Administration: ACC; Funding Acquisition: ACC.

\section{Sources of funding}

The authors were supported by grants from the Ministry of Education (grant numbers MOE2013-T2-2-054 and MOE2017-T2-1-089) awarded to ACC. TT was supported by a Singapore International Graduate Award from the A*STAR Graduate Academy. $\mathrm{HC}$ was supported by grants MOE-2016-T2-1-001 from the Singapore Ministry of Education and NMRC-CG-M009 from the National Medical Research Council. The authors received additional support from Duke-NUS Medical School, a Biomedical Research Council block grant to the Institute of Molecular and Cell Biology, and grants from the A*STAR Joint Council Office (grant numbers 1231AFG030 and 1431AFG120) awarded to ACC.

\section{Acknowledgements}

We thank Joses Ho, other members of the lab and TT's thesis committee for helpful feedback. We thank Jessica Tamanini of Insight Editing London for a critical reading of the manuscript before submission. 


\section{Methods}

\section{Drosophila strains}

Flies were raised on fly medium (Temasek Life Sciences Laboratories, 2018) at $25^{\circ} \mathrm{C}$ in a $12 \mathrm{~h}$ light: $12 \mathrm{~h}$ dark cycle. For optogenetic experiments, the flies were kept in the dark and reared on fly food supplemented with $0.5 \mathrm{mM}$ all-trans-retinal (Sigma-Aldrich, USA) for 2 days prior to experimentation. For starvation experiments, the flies were reared on $2 \%$ agarose for $12-18 \mathrm{~h}$ prior to the assay. Wild type flies were cantonized $w^{1118}$; all the ORx-Gal4 and UAS-CsChrimson strains were obtained from the Bloomington Drosophila Stock Center (USA) (Table S5) (Couto et al., 2005; Dobritsa et al., 2003; Klapoetke et al., 2014; Vosshall et al., 2000).

\section{Optogenetic preference assay}

The wind- and light-induced self-administration response (WALISAR) assay was conducted as follows. Two rectangular assemblies $(11.5 \times 14.5 \times 0.3 \mathrm{~cm})$ were cut from acrylic sheets; each assembly contained 26 chambers $(50 \times 4 \mathrm{~mm})$, herein referred to as WALISAR chambers. Airflow inlets and outlets were milled into the ends of each chamber. Optogenetic illumination was achieved using LEDs [LUXEON Rebel LEDs on a SinkPAD-II $10 \mathrm{~mm}$ Square Base; red $(617 \mathrm{~nm})$, green $(530 \mathrm{~nm})$, blue $(470 \mathrm{~nm})$, each equipped with lenses ( $17.7^{\circ} 10 \mathrm{~mm}$ Circular Beam Optic)] and attached to heatsinks located above the arena on both sides at $\mathrm{a} \sim 45^{\circ}$ angle. The LEDs were grouped by color and were powered by $700 \mathrm{~mA}$ BuckPuck drivers. Custom instrumentation software (CRITTA) was used to control the intensity and timing of the LEDs throughout the experiments. To achieve a half-dark/half-lit arena for the optogenetic choice experiments, two black acrylic shields were placed between the arena and the LEDs on each side and were adjusted to cast shade on either half of the chamber. By switching the LEDs on either side, the half of the arena that was lit could be alternated. The temperature difference between the dark and lit halves of the WALISAR chambers that could arise from the LED illumination was 
measured for the duration of a whole experiment using thermocouples, and found to be a negligible $\sim 0.3^{\circ} \mathrm{C}$ (https://doi.org/10.5281/zenodo.4545940). Compressed air was connected to the airflow inlets of the arena, with an intervening stopcock valve (Cole-Parmer) to modulate the flow rate. The air flow in all experiments using wind was 35 $\mathrm{cm} / \mathrm{s}$, as described in a previous study (Bell and Wilson, 2016). The airflow was measured before every experiment with an airflow meter (Cole-Parmer).

Flies were collected 2-3 days before experiments and cold anesthesia was administered for 20-30 s, just prior to their transfer into the WALISAR chambers. A single experimental cycle consisted of: acclimatization of the flies for $30 \mathrm{~s}$; illumination of the left half of the arena for $45 \mathrm{~s}$; no illumination for $30 \mathrm{~s}$; illumination of right half of the arena for $45 \mathrm{~s}$; and no illumination for $30 \mathrm{~s}$. Each group of flies was tested in six conditions comprising three light intensities $\left(14,42,70 \mu \mathrm{W} / \mathrm{mm}^{2}\right)$, each with and without wind $(35 \mathrm{~cm} / \mathrm{s})$ (on/off), totaling 12 steps (S1-S12) (Figure S1A). The light intensities were measured with a thermal power sensor (Thorlabs S310C) connected to a power and energy-meter console (Thorlabs PM100D). The flies were recorded with an AVT Guppy PRO F046B camera fitted with an IR bandpass filter, which was positioned on the top of the arena. The camera was connected to a computer running custom LabVIEW software (CRITTA), which was used to determine the flies' head positions.

\section{WALISAR protocol and validation}

Given that the ordering of the experiments in repeated-measure designs can affect outcomes, the WALISAR protocol was tested with two sets of experiments performed on Orco-Gal4 > UAS-CsChrimson flies, in an ascending or descending light-intensity order (Collie et al., 2003; Howitt and Cramer, 2007; McCall and Appelbaum, 1973). The responses in the two orders were similar for eight of the 12 epochs, being different only for S2, S3, S10, and S11. The difference was due to the weak valence responses in the second and third order epochs: S2 and S3 
produced lower effects in the ascending order (Figure S4E), S10 and S11 produced lower effects in the descending order (Figure S4F). The underestimation of the second and third order epochs had little effect on the overall interpretation of the results because eight of the epochs generated similar results. Moreover, even a hypothetical extreme case in which the valence is overlooked in the second and third order epochs would result in a false negative, rather than a false positive. As such, the experimental order was concluded to not have a major effect on the WALISAR results. Additionally, the effect sizes in the first epoch tended to be smaller than the second epoch when the light was downwind of the air flow (Figure S4); to eliminate this bias, only second epochs were used for further data analysis.

One concern is that the activity rates and temporal structure of optogenetic stimulation are likely different from direct odor stimulation. To mitigate this, we conducted a study of the effect of optogenetic temporal structure, finding that-while this is a relevant concern-continuous illumination is a more conservative method (Tumkaya et al., 2019). We also benchmarked behavioral responses for the Orco neurons against results from a prior study that performed physiological recordings and used a different temporal structure (Bell and Wilson, 2016), finding that the WALISAR protocol has comparable sensitivity (Figure S3).

\section{WALISAR data analysis: the wTSALE metric}

Fly-position data were analyzed with custom Python scripts. The valence of each fly was measured in terms of how much time it spent in the light after first encountering one of the lit zones. Specifically, the first frame in which a fly entered the lit zone was considered the start of the test session; after this initial light encounter, the amount of time spent in the dark was subtracted from time spent in the light and finally divided by the total amount of time. This metric is designated 'Time Spent After Light Encounter' (TSALE). The duration between the first light 
discovery and the end of the light epoch varied across individual files, from never discovering the light $(0 \mathrm{~s})$ to being exposed to the light at the start of the epoch (60 s). As such, each fly's TSALE score was weighted by the post-light duration, termed 'weighted-TSALE' (wTSALE). This weighting was achieved by multiplying the TSALE with the ratio between the remaining time and the full duration of the test epoch. The wTSALE score was calculated for each fly and then averaged for the control and test genotypes. To calculate the effect sizes, responder and driver controls were pooled into a single control group. The mean difference $(\Delta)$ between the pooled-control and test groups was taken as the effect size ( $\triangle$ wTSALE). The $\Delta$ distributions and $95 \%$ bootstrapped confidence intervals (Cls) were calculated using the DABEST Python package (Ho et al., 2019), and presented in the results in the following format: " $\Delta[95 \mathrm{Cl}$ lower bound, upper bound]."

\section{WALISAR data analysis by Empirical Bayes method}

To draw conclusions from multiple experiments (performed at one of three light intensities) for a given ORN, the dimension of the data was reduced into one summary statistic $(D)$ for each ORN, as follows (Efron et al., 2001):

$D_{i}=M_{\text {exp }}-\left(\left(M_{c t r l 1}+M_{c t r l 2}\right) * 0.5\right)$,

where $M$ is the mean of the wTSALE score for experimental and control flies. Then, $D$ was used to calculate a $Z$ score:

$Z_{i}=D_{i} /\left(\alpha_{0}+S_{i}\right)$

where $D_{i}$ is the effect size of each ORN, $S_{i}$ is the standard deviation, and $\alpha_{0}$ is $90^{\text {th }}$ percentile of all the $S$ values.

After calculating a vector of $Z$ scores, the EBprot software package (Koh et al., 2015) was used to implement the Empirical Bayes (EB) method to differentiate true behavioural changes from noisy observations, and to compute the posterior probability of each effect size and 
associated false discovery rate (FDR). The signed probabilities indicate the likelihood that the valence scores came from each of the three candidate distributions (negative, neutral, and positive valence). Prior to calculating the signed probabilities, EBprot removes outliers from the valence distributions (Koh et al., 2015). Effect sizes associated with a $<25 \%$ FDR were considered for further analysis.

\section{Modeling the valence responses of ORN combinations}

The ORN-combo valences were modeled using three functions, each widely used in neural network research for input pooling: summation, max-pooling, and min-pooling. Summation simply sums two individual valence values, while max-pooling and min-pooling return the larger or smaller absolute value of the two components, respectively (Goodfellow et al., 2016), as follows:

Summation $=\left(i_{0}+i_{1}\right)$

Max - pooling $=\max \left(i_{0}, i_{1}\right.$, key $=$ absolute value $)$

Min - pooling $=\min \left(i_{0}, i_{1}\right.$, key $=$ absolute value $)$

where $i_{0}$ and $i_{1}$ are real numbers.

For example, given $i_{0}=-2$ and $i_{1}=+1$, the summation, max-pooling, and min-pooling functions would return -1, -2 , and +1 , respectively.

\section{Correlation and agreement analyses}

Linear regressions were performed using the SciPy library in Python (Jones et al., 2001). Bootstrapped Cls for the coefficient of determination $\left(R^{2}\right)$ were calculated using the scikits-bootstrap package in Python (Evans, 2019). Bland-Altman plots were generated using custom scripts using the matplotlib and seaborn libraries in Python (Bland and Altman, 1999; Hunter, 2007; Waskom et al., 2017). 


\section{ORN interaction analyses}

Custom R scripts were used to perform statistical inference on multiple linear regression models characterizing the association between single-ORN classes and ORN-combo. Single-ORNs (ORN1 and ORN2) were used as predictors, while the ORN-combo phenotype was the dependent variable, as follows:

ORN - combo $=\beta_{0}+\beta_{1} \times$ ORN $1+\beta_{2} \times$ ORN 2

From the original data, 10,000 bootstrap samples of the single-fly data were drawn from each group (ORN1, ORN2, and ORN-combo) and the flies were ordered by their valence levels and paired into trios of ORN1, ORN2, and ORN-combo. In each bootstrap sample, a multiple linear regression model was fitted, which revealed the probabilistic distributions of the beta weights $\left(\beta_{1}\right.$ and $\left.\beta_{2}\right)$.

\section{Predictive modeling}

We considered that the available published data has a disproportionate ORN to odor ratio (23 to 110), with the possibility of internal correlation. Prior to building a valence prediction model, we performed exploratory analyses on the datasets: Pearson correlation among the ORNs, hierarchical clustering, and partial least squares discriminant analysis (PLS-DA). All three analyses were conducted using the pandas and scikit-learn libraries in Python (McKinney, 2010; Pedregosa et al., 2011). The eight latent variables (LVs) calculated by the PLS-DA analysis were used to train the linear and non-linear models using the scikit-learn library in Python (Pedregosa et al., 2011). The performance of the models were evaluated by using the adjusted- $\mathrm{R}^{2}$ and the root-mean-squared error (RMSE). The RMSE values were calculated using a shuffled-split ten-fold cross-validation scheme in Python's scikit-learn library (Pedregosa et al., 2011). To weight the prediction models, we calculated the Pearson correlation coefficients between Hallem and Carlson's firing rates and our single-ORN wTSALE valence scores of the 23 ORNs for all 
110 samples. So that the odors with higher correlation play a more important role in the model fitting, absolute value of these correlations were then used to re-weight the odor samples in the regression model.

\section{Re-analysing previous valence studies}

The relevant studies were downloaded in pdf format, and the data of interest were extracted by using the measuring tool in Adobe Acrobat Pro (Adobe Systems USA). The extracted values for control and experimental groups were then used to calculate the standardized effect size, Cohen's $d$ (Cumming and Calin-Jageman, 2016). If technical replicates were used to calculate statistics, the effect sizes were corrected accordingly (Table S4).

\section{Data and code availability}

All of the data generated by this study are available to download from Zenodo

(https://doi.org/10.5281/zenodo.3994033). The code is available at https://github.com/ttumkaya/WALiSuite_V2.0. 


\section{Supplementary material}

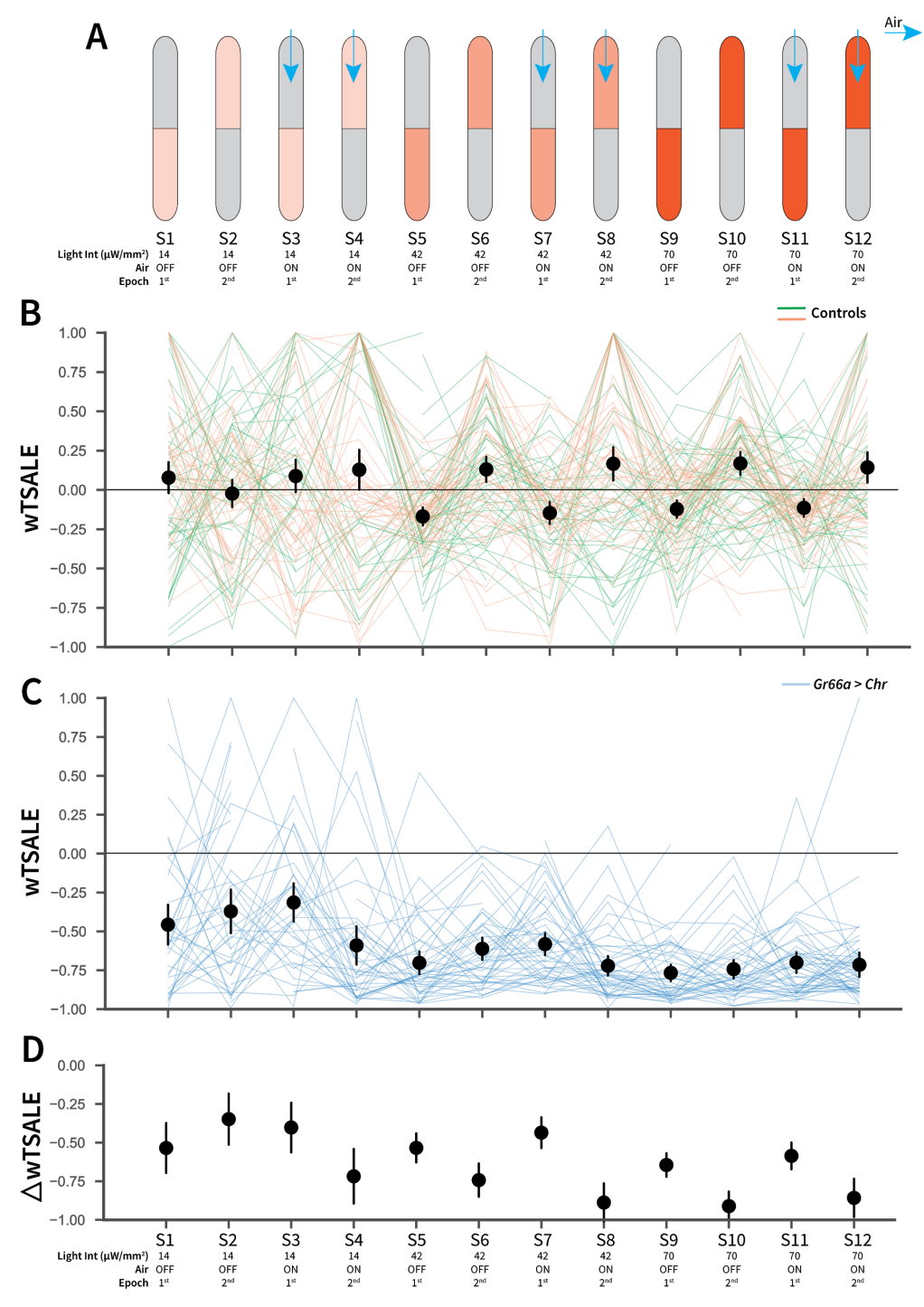

Figure S1. An optogenetic behavior assay reports on Gr66a-induced avoidance

A. In each experiment, the flies were subjected to 12 consecutive epochs: three light intensities that were applied in an increasing order $\left(14,42,70 \mu \mathrm{W} / \mathrm{mm}^{2}\right)$; with and without airflow; and the illumination side was flipped in alternating epochs.

B. The light preferences of control flies across the 12 epochs, as estimated by weighted time spent after light encounter (wTSALE). Each line represents a single fly; the green lines indicate $w^{1118}$; Gr66a-Gal4 flies, and the orange lines indicate $w^{1118}$; UAS-CsChrimson flies. The black dots represent the mean wTSALE scores of all control flies per condition; the whiskers indicate the $95 \% \mathrm{Cls}$.

C. The light preference of Gr66a-Gal4 > UAS-CsChrimson flies across the epochs.

D. The optogenetic effect sizes, calculated by taking the difference of test and control preferences ( $\triangle \mathrm{wTSALE}$ ). 

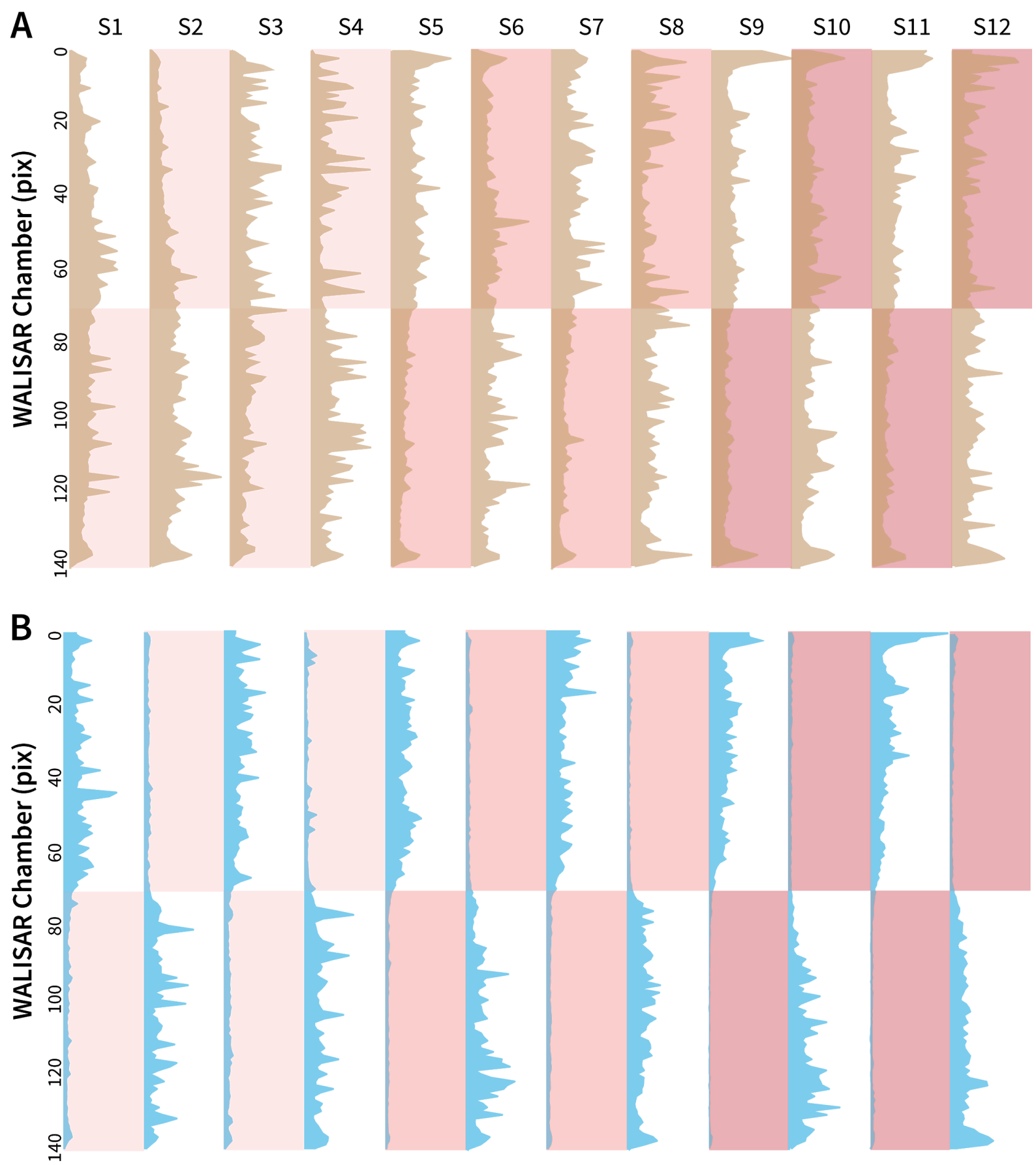

Figure S2. Occupancy analysis of the WALISAR chambers during Gr66a-neuron activation

A. The number of occurrences of the control flies ( $w^{1118}$; Gr66a-Gal4 and $w^{1118}$;

UAS-CSChrimson, $\mathrm{N} \cong 104$ ) across the WALISAR chambers are drawn. The brown curves present the total number, the $y$ axis represents the chamber, and the $x$ axis is the frequency of occurrence (ranging between 0 and 2,000). The red shading indicates the three light intensities; and the labels from S1 to S12 represent the 12 experimental epochs (Figure 1C).

B. The number of occurrences of the experimental flies (Gr66a-Gal4>UAS-CsChrimson, $\mathrm{N} \cong$ 52) across the WALISAR chambers are summed and drawn for the 12 epochs. The blue 
curves represent the total number, the $y$ axis represents the chamber, and the $x$ axis is the frequency of occurrence (ranging between 0 and 1,500).

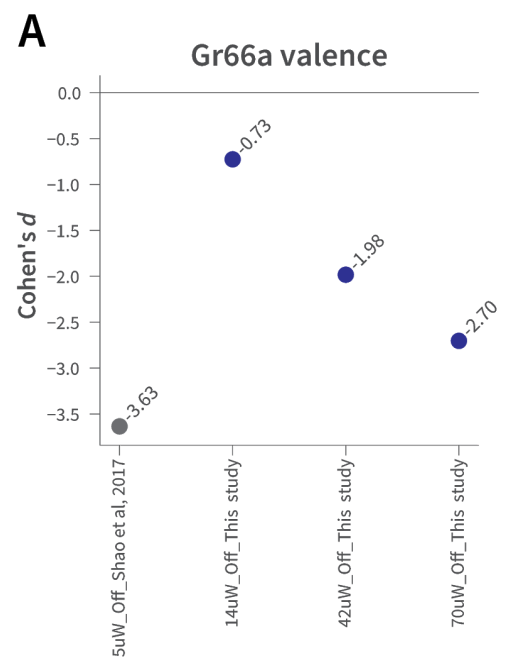

B

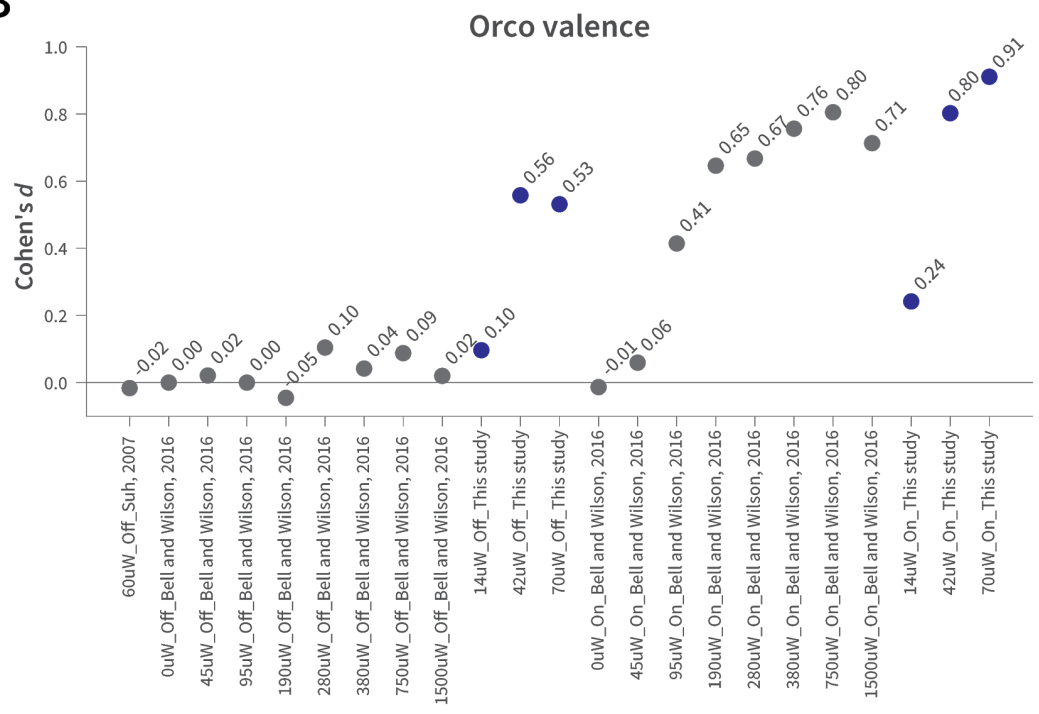

Figure S3. The effect sizes in WALISAR and previously reported optogenetic valence assays are comparable

A. A comparison of the Gr66a-mediated aversion (Cohen's $d$ ) between this study at different light intensities and Shao et al. at $5 \mu \mathrm{W} / \mathrm{mm}^{2}$ of blue light, both in still-air conditions (Shao et al., 2017).

B. The magnitude of the attraction (standardized effect size, Cohen's $d$ ) produced by Orco-neuron activation in the present study is compared to that of two previous studies (Bell and Wilson, 2016; Suh et al., 2007). The light intensity is shown in $\mu \mathrm{W} / \mathrm{mm}^{2}$, while "On" and "Off" indicate the presence of wind. 

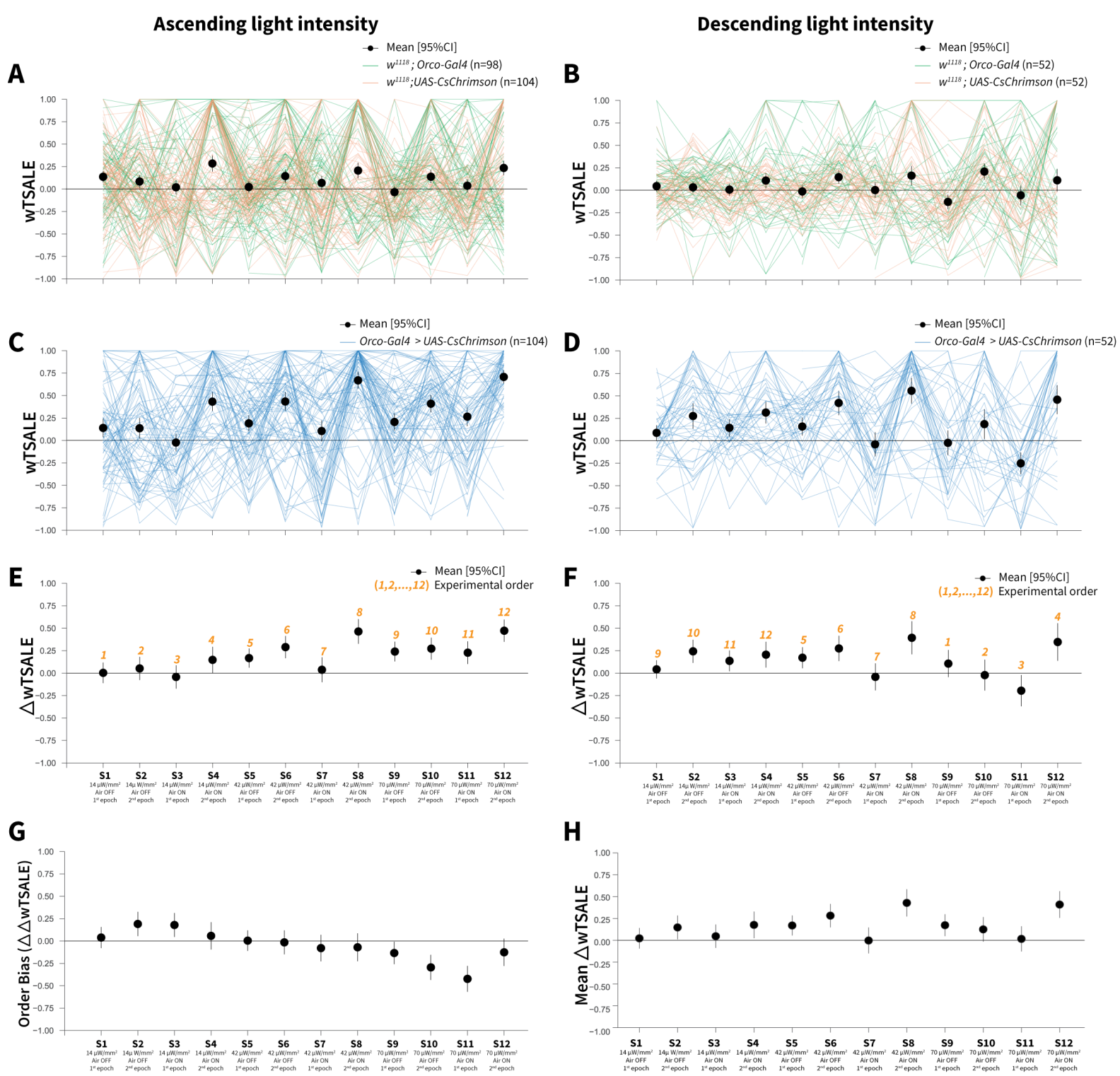

Figure S4. Orco-neuron activation triggers attraction regardless of the experimental order

A. The light preference of control flies and the mean wTSALE differences across 12 epochs, in which the light intensities were applied in an ascending order. The green lines represent $w^{1118}$; Orco-Gal4 flies, and the orange lines represent $w^{1118} ;$ UAS-CsChrimson flies. The black dots represent the mean wTSALE scores (with 95\% Cls) per epoch.

B. The light preference of the control flies in which the light intensities were applied in a descending order. The green lines represent $w^{1118}$; Orco-Gal4 flies, and the orange lines represent $w^{1118}$; UAS-CSChrimson flies. The black dots represent the mean wTSALE scores (with 95\% Cls) per epoch.

C. The light preference of the test flies in which the light intensities were applied in an ascending order. The blue lines represent Orco-Gal4 > UAS-CsChrimson flies.

D. The test flies' light preference was presented across the 12 epochs. The blue lines represent Orco-Gal4 > UAS-CSChrimson flies. 
E. The mean wTSALE differences between the test and combined-control flies across the 12 conditions, in ascending order. The orange numbers indicate the order of the respective experiment. The flies were attracted to activity in the Orco+ neurons in the absence of wind.

F. The mean wTSALE differences between the test and combined-control flies across 12 conditions in the descending order. The flies were attracted to activity in the Orco+ neurons in the absence of wind.

G. A comparison plot of the effect sizes from experiments in which the light intensities were applied in an ascending or descending order. The black dots represent the difference of the mean $\triangle W T S A L E$ differences ( $\triangle \triangle W T S A L E$ ) from the two differently ordered experiments.

H. The mean effect sizes of the ascending and descending light-intensity experiments across the epochs. The black dots represent the mean of the $\triangle$ WTSALE differences ( $\triangle$ WTSALE) from the two differently ordered experiments along with $95 \% \mathrm{Cls}$. 

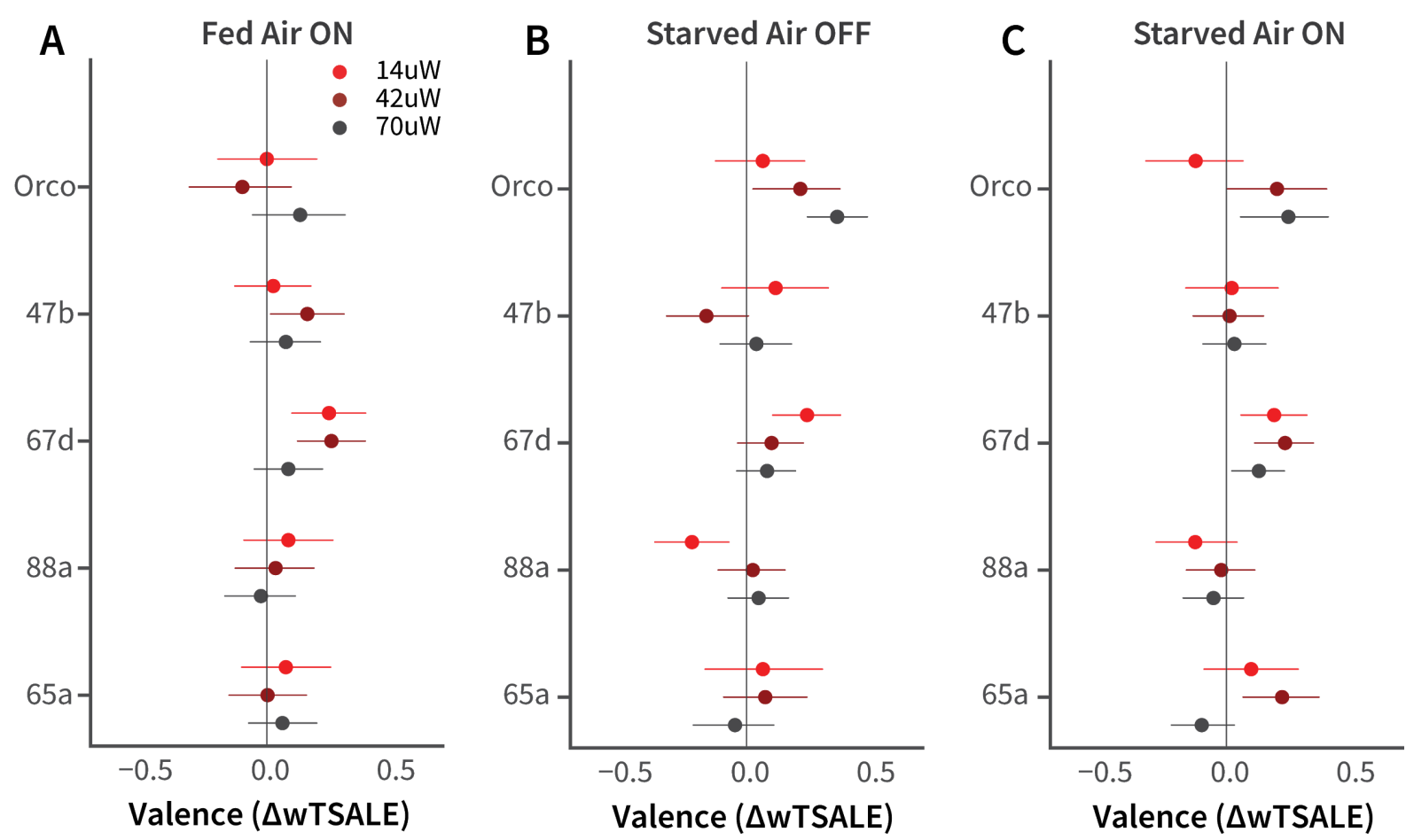

Figure S5. Female-fly responses to activation of Orco and four pheromone-related cells

A-C. The olfactory valence of the Orco and four single-ORN types were tested in fed or starved female flies with or without airflow. The valence responses are represented as the mean difference $(\triangle \mathrm{WTSALE})$ of control $(\mathrm{N} \cong 104)$ and test $(\mathrm{N} \cong 52)$ flies, along with $95 \% \mathrm{Cls}$. 

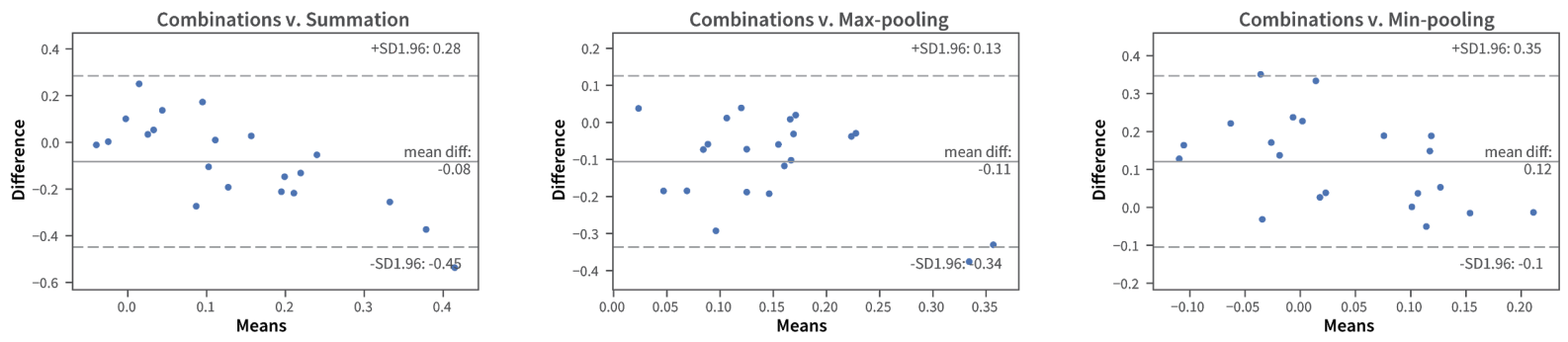

Figure S6. Agreement analysis of the observed and predicted ORN-combo valence responses by three pooling functions

A-C. Bland-Altman plots of ORN-combo valence responses and predictions by the (A) summation, (B) max-pooling, and (C) min-pooling models. Even though the mean difference of the two tested values ( $y$ axis) is low in all models, the limits of agreement (SD $-1.96,+1.96)$ are sufficiently wide for them to be considered dissimilar. 


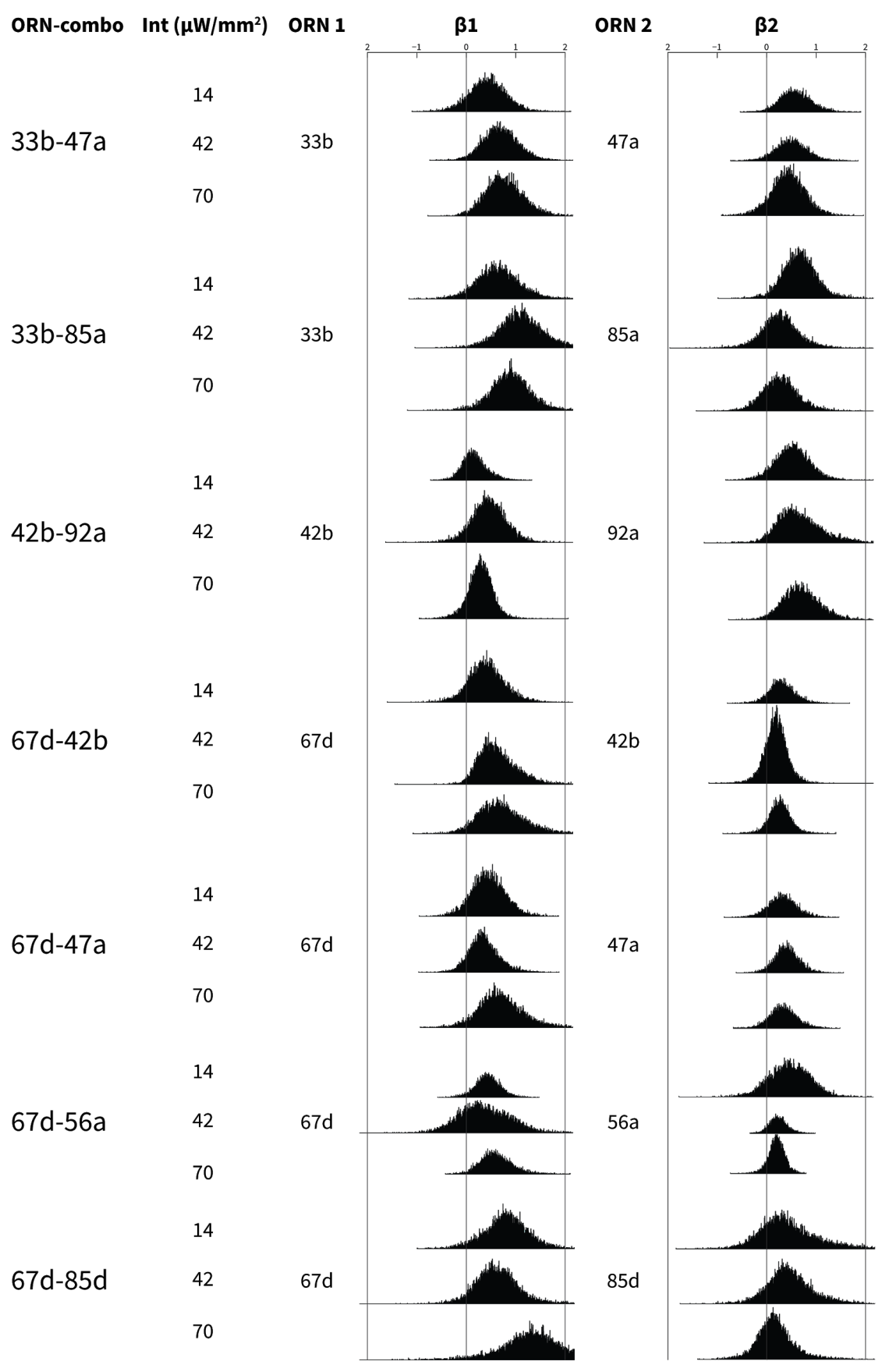

Figure S7. Bootstrapped distributions of $\boldsymbol{\beta}$ weights of the ORN-combo constituents Associations between ORN-combos and their constituent single-ORN types are tested by using a multiple linear regression analysis approach. ORN1 and ORN2 columns indicate the odor receptor types that are used to generate the respective ORN-combo. The light intensity used in the experiments are shown in the intensity (Int; $\mu \mathrm{W} / \mathrm{mm}^{2}$ ) column. $\beta 1$ and $\beta 2$ columns present the bootstrapped $\beta$ distributions of the ORN1 and ORN2, respectively; the y axes indicate the count, and are all plotted using the same scale. 


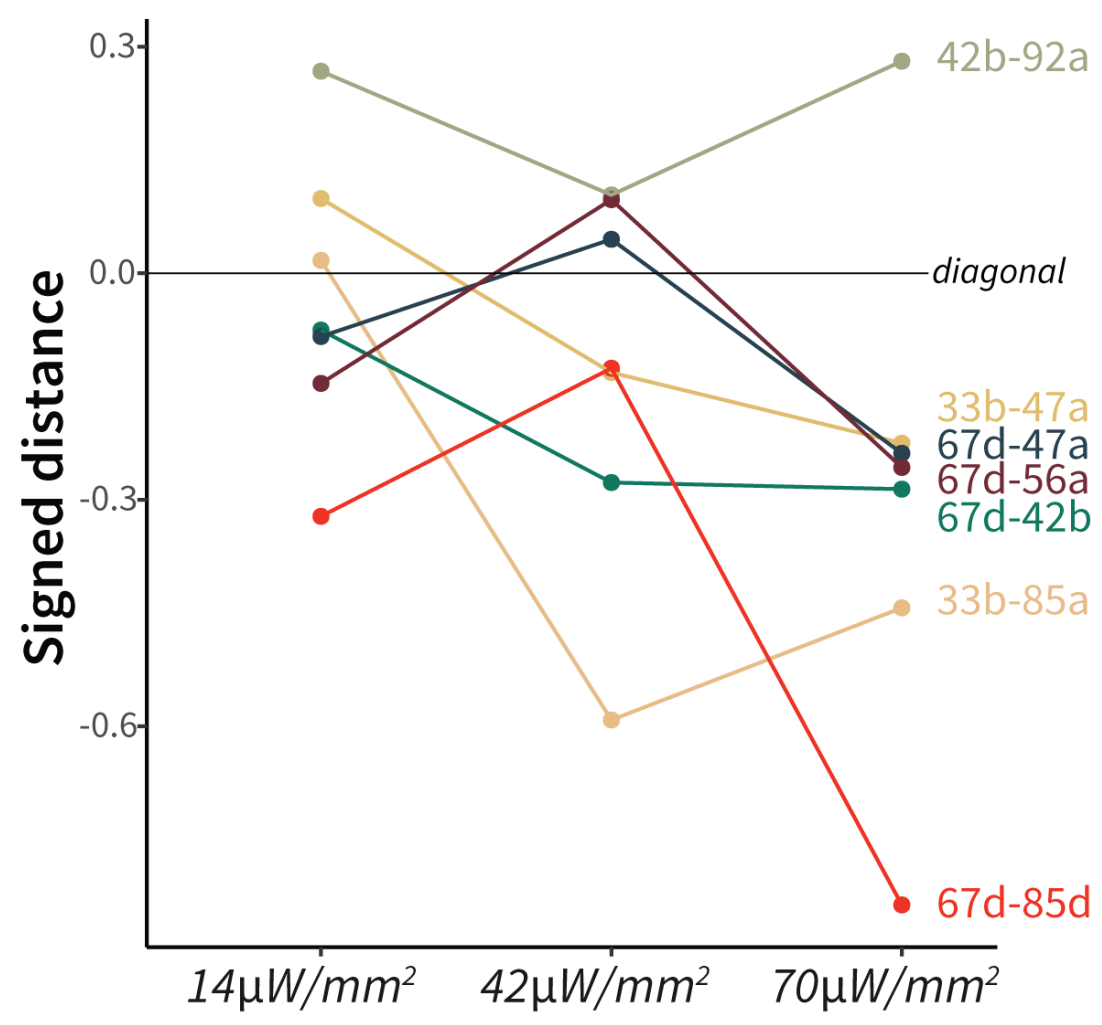

Figure S8. Signed distances of the ORN-combos from the diagonal line

The $\beta$ weights of the ORN-combos were modelled by multiple linear regression. Here, the signed distances of each ORN-combo from the diagonal line over three light intensities are drawn, showing that ORN weightings change magnitude and, in some cases, the dominant partner changes with increasing optogenetic stimulus. 

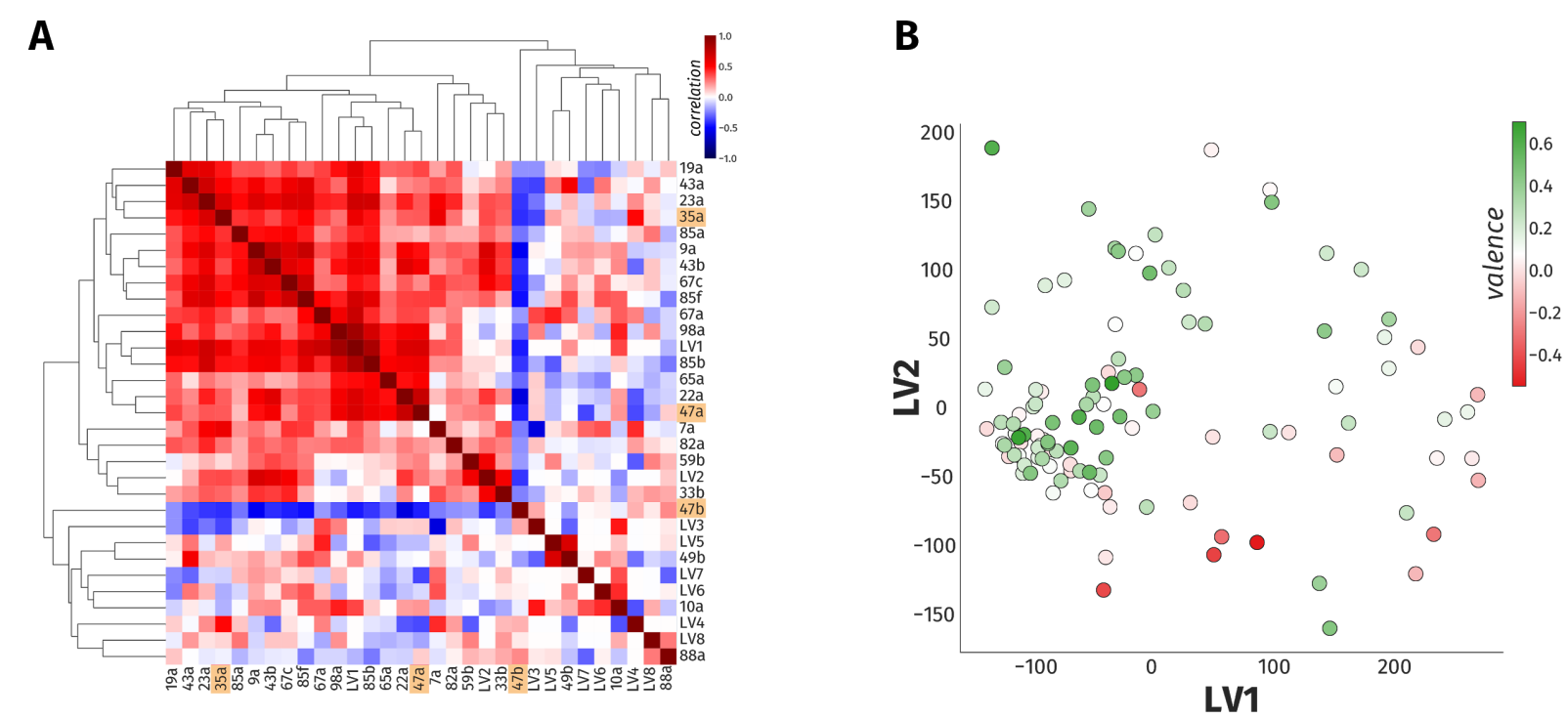

Figure S9. A linear model accounts for only $23 \%$ of the variance in odor behavior

A. The heatmap of hierarchical clustering shows the $27 \times 27$ Pearson correlation coefficients among the 23 ORN types and eight LVs from PLS-DA analysis. The internal correlation of LVs and their constituent ORN-types is indicated by color, where blue and red ends of the spectrum represent negative and positive correlations, respectively. The three ORN types that produced a valence response in the WALISAR screen are highlighted in orange.

B. A scatter plot displays projection of 110 odorants to two-dimensional LV space. The valence of the odorants are shown with a color gradient ranging from red to green, indicating aversive and attractive odorants, respectively. 
A

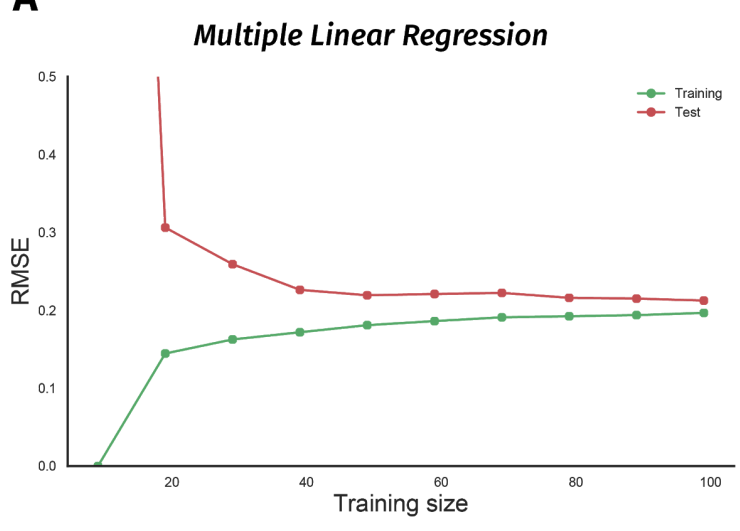

C

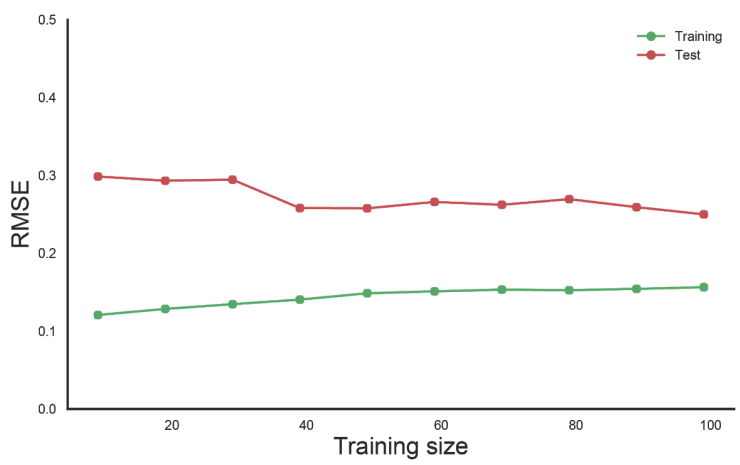

B

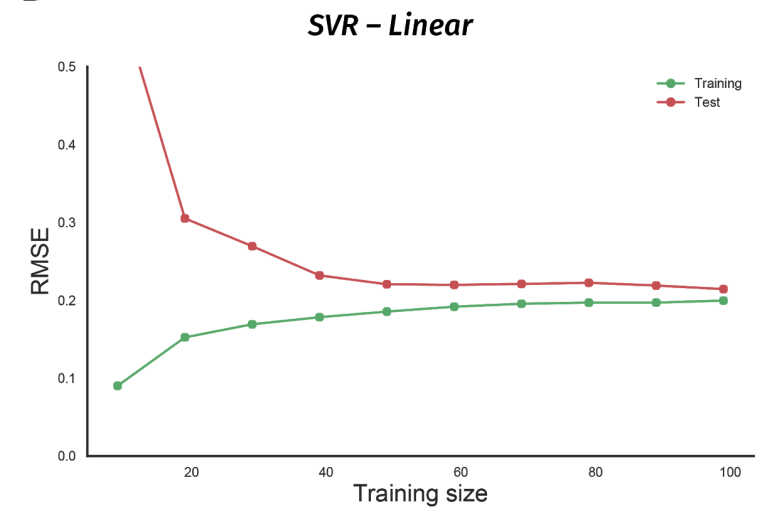

D SVR-Radial Basis Function

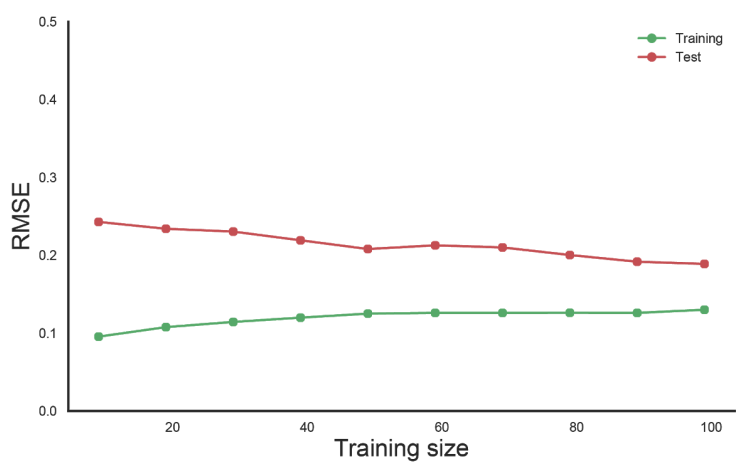

Figure S10. Non-linear models suffer from the small size of the odor-valence data set

A. Performance of a multiple linear regression (MLR) model is shown as the training data size is incrementally increased. The y-axis indicates the root-mean-squared-error (RMSE), while the $x$-axis is the size of the training data-set. The green and red traces in all the panels represent error rates on training and 10 -fold cross-validation test data, respectively.

B. Learning curve of a support vector regression (SVR) model with a linear kernel is drawn. Both MLR and SVR linear models show convergent learning curves.

C. Performance of a support vector regression (SVR) model using a non-linear, polynomial kernel is plotted over an increasing training data-set size.

D. Error rates of a support vector regression (SVR) model with a non-linear, radial-basis-function kernel are plotted over a growing training data size. Both nonlinear models' learning curves fail to converge, indicating overfit. 


\section{Table S1. ORN types with known innate valence.}

The innate responses of 24 types of ORNs that have been reported at time of writing (January, 2020). The valence response, experimental assay, stimulus type, sex, and the developmental stage of the flies varied across studies. The valence column shows the response direction produced by the respective ORN: negative $(-)$, positive $(+)$, or indifferent (o). The assay column presents the nature of the assay used in the experiments: oviposition (place preference for egg-laying in female flies), two-choice (any assay by which the flies are presented with a choice to activate the ORN), locomotor (the assay in which motor behavior of the larvae is used to deduce valence). The stimulus column shows the type of the stimulus applied on the ORN: olfactogenetics (geosmin on ORNs that ectopically express the Or56a receptor), optogenetic (light stimulus on genetically modified ORNs), odor. In the sex column, F, M, M/F, and N/A indicate female, male, both male and female, and information that is not available, respectively. The stage column presents the developmental stage of the animals used in the experiment: larva, adult, and both (larva and adult).

Receptor Valence Assay

\begin{tabular}{|c|c|c|c|c|c|c|}
\hline Or7a & $\begin{array}{l}- \\
0\end{array}$ & $\begin{array}{l}\text { oviposition } \\
\text { two-choice }\end{array}$ & $\begin{array}{l}\text { olfactogenetics } \\
\text { optogenetic }\end{array}$ & $\begin{array}{l}F \\
M\end{array}$ & $\begin{array}{l}\text { adult } \\
\text { adult }\end{array}$ & $\begin{array}{l}\text { (Chin et al., 2018) } \\
\text { this study }\end{array}$ \\
\hline & + & oviposition & odor & $\mathrm{F}$ & adult & (Dweck et al., 2013) \\
\hline \multirow[t]{4}{*}{ Or19a } & 0 & oviposition & olfactogenetics & $\mathrm{F}$ & adult & (Chin et al., 2018) \\
\hline & 0 & two-choice & optogenetic & M & adult & this study \\
\hline & + & two-choice & odor & $M / F$ & adult & (Knaden et al., 2012) \\
\hline & + & two-choice & odor & $\mathrm{F}$ & adult & (Semmelhack and Wang, 2009) \\
\hline \multirow[t]{4}{*}{ Or22a } & - & two-choice & odor & $\mathrm{F}$ & adult & (Gao et al., 2015) \\
\hline & + & two-choice & optogenetic & M & adult & (Bell and Wilson, 2016) \\
\hline & 0 & oviposition & olfactogenetics & $\mathrm{F}$ & adult & (Chin et al., 2018) \\
\hline & 0 & two-choice & optogenetic & M & adult & this study \\
\hline \multirow[t]{2}{*}{ Or23a } & 0 & oviposition & olfactogenetics & $\mathrm{F}$ & adult & (Chin et al., 2018) \\
\hline & 0 & two-choice & optogenetic & M & adult & this study \\
\hline \multirow[t]{4}{*}{ Or35a } & 0 & oviposition & olfactogenetics & $\mathrm{F}$ & adult & (Chin et al., 2018) \\
\hline & + & two-choice & optogenetic & M & adult & this study \\
\hline & + & locomotor & optogenetic & NA & larva & (Hernandez-Nunez et al., 2015) \\
\hline & + & two-choice & odor & NA & larva & (Mathew et al., 2013) \\
\hline \multirow[t]{2}{*}{ Or42a } & 0 & two-choice & odor & $\mathrm{F}$ & adult & (Jung et al., 2015) \\
\hline & + & two-choice & optogenetic & M & adult & (Bell and Wilson, 2016) \\
\hline
\end{tabular}




\begin{tabular}{|c|c|c|c|c|c|c|}
\hline & \multirow{3}{*}{$\begin{array}{l}0 \\
0 \\
0\end{array}$} & \multirow{3}{*}{$\begin{array}{l}\text { oviposition } \\
\text { two-choice } \\
\text { two-choice }\end{array}$} & \multirow{3}{*}{$\begin{array}{l}\text { olfactogenetics } \\
\text { olfactogenetics } \\
\text { optogenetic }\end{array}$} & \multirow{3}{*}{$\begin{array}{l}\mathrm{F} \\
\mathrm{F} \\
\mathrm{M}\end{array}$} & \multirow{2}{*}{$\begin{array}{l}\text { adult } \\
\text { adult }\end{array}$} & \multirow{3}{*}{$\begin{array}{l}\text { (Chin et al., 2018) } \\
\text { (Chin et al., 2018) } \\
\text { this study }\end{array}$} \\
\hline & & & & & & \\
\hline & & & & & adult & \\
\hline \multirow{7}{*}{ Or42b } & + & two-choice & odor & $\mathrm{F}$ & adult & (Semmelhack and Wang, 2009) \\
\hline & + & two-choice & odor & NA & larva & (Mathew et al., 2013) \\
\hline & + & two-choice & odor & $\mathrm{F}$ & adult & (Gao et al., 2015) \\
\hline & 0 & two-choice & odor & $\mathrm{F}$ & adult & (Jung et al., 2015) \\
\hline & + & two-choice & optogenetic & M & adult & (Bell and Wilson, 2016) \\
\hline & 0 & oviposition & olfactogenetics & $\mathrm{F}$ & adult & (Chin et al., 2018) \\
\hline & + & two-choice & optogenetic & M & adult & this study \\
\hline \multirow[t]{2}{*}{ Or43a } & 0 & oviposition & olfactogenetics & $\mathrm{F}$ & adult & (Chin et al., 2018) \\
\hline & 0 & two-choice & optogenetic & M & adult & this study \\
\hline \multirow[t]{2}{*}{ Or47a } & 0 & oviposition & olfactogenetics & $\mathrm{F}$ & adult & (Chin et al., 2018) \\
\hline & + & two-choice & optogenetic & M & adult & this study \\
\hline \multirow[t]{2}{*}{ Or47b } & - & oviposition & olfactogenetics & $\mathrm{F}$ & adult & (Chin et al., 2018) \\
\hline & + & two-choice & optogenetic & M & adult & this study \\
\hline \multirow[t]{3}{*}{ Or49a } & - & oviposition & olfactogenetics & $\mathrm{F}$ & adult & (Chin et al., 2018) \\
\hline & 0 & two-choice & optogenetic & M & adult & this study \\
\hline & - & two-choice & odor & NA & adult & (Stensmyr et al., 2012) \\
\hline \multirow[t]{2}{*}{ Or56a } & + & two-choice & optogenetic & M & adult & (Bell and Wilson, 2016) \\
\hline & - & two-choice & optogenetic & M & adult & this study \\
\hline \multirow[t]{2}{*}{ Or59c } & - & oviposition & olfactogenetics & $\mathrm{F}$ & adult & (Chin et al., 2018) \\
\hline & - & two-choice & optogenetic & M & adult & this study \\
\hline \multirow[t]{3}{*}{ Or65a } & 0 & oviposition & olfactogenetics & $\mathrm{F}$ & adult & (Chin et al., 2018) \\
\hline & 0 & two-choice & optogenetic & M & adult & this study \\
\hline & + & two-choice & odor & NA & larva & (Mathew et al., 2013) \\
\hline \multirow[t]{3}{*}{ Or67b } & + & two-choice & optogenetic & M & adult & (Bell and Wilson, 2016) \\
\hline & - & oviposition & olfactogenetics & $\mathrm{F}$ & adult & (Chin et al., 2018) \\
\hline & 0 & two-choice & optogenetic & M & adult & this study \\
\hline \multirow[t]{3}{*}{ Or67d } & 0 & oviposition & olfactogenetics & $\mathrm{F}$ & adult & (Chin et al., 2018) \\
\hline & + & two-choice & optogenetic & M & adult & this study \\
\hline & - & oviposition & olfactogenetics & $\mathrm{F}$ & adult & (Chin et al., 2018) \\
\hline \multirow[t]{2}{*}{ Or71a } & 0 & two-choice & olfactogenetics & $\mathrm{F}$ & adult & (Chin et al., 2018) \\
\hline & 0 & two-choice & optogenetic & M & adult & this study \\
\hline \multirow[t]{3}{*}{ Or82a } & - & oviposition & olfactogenetics & $\mathrm{F}$ & adult & (Chin et al., 2018) \\
\hline & 0 & two-choice & optogenetic & M & adult & this study \\
\hline & + & two-choice & odor & $M / F$ & adult & (Ronderos et al., 2014) \\
\hline
\end{tabular}




\begin{tabular}{|c|c|c|c|c|c|c|}
\hline Or83c & $\begin{array}{l}- \\
+\end{array}$ & $\begin{array}{l}\text { oviposition } \\
\text { two-choice }\end{array}$ & $\begin{array}{l}\text { olfactogenetics } \\
\text { optogenetic }\end{array}$ & $\begin{array}{l}\mathrm{F} \\
\mathrm{M}\end{array}$ & $\begin{array}{l}\text { adult } \\
\text { adult }\end{array}$ & $\begin{array}{l}\text { (Chin et al., 2018) } \\
\text { this study }\end{array}$ \\
\hline \multirow{7}{*}{ Or85a } & + & two-choice & odor & $M / F$ & adult & (Knaden et al., 2012) \\
\hline & - & two-choice & odor & $\mathrm{F}$ & adult & (Semmelhack and Wang, 2009) \\
\hline & - & two-choice & odor & $\mathrm{F}$ & adult & (Gao et al., 2015) \\
\hline & + & two-choice & optogenetic & M & adult & (Bell and Wilson, 2016) \\
\hline & - & oviposition & olfactogenetics & $\mathrm{F}$ & adult & (Chin et al., 2018) \\
\hline & 0 & two-choice & olfactogenetics & $\mathrm{F}$ & adult & (Chin et al., 2018) \\
\hline & 0 & two-choice & optogenetic & M & adult & this study \\
\hline \multirow[t]{2}{*}{ Or85d } & - & oviposition & olfactogenetics & $\mathrm{F}$ & adult & (Chin et al., 2018) \\
\hline & - & two-choice & optogenetic & M & adult & this study \\
\hline \multirow[t]{3}{*}{ Or88a } & 0 & oviposition & olfactogenetics & $\mathrm{F}$ & adult & (Chin et al., 2018) \\
\hline & 0 & two-choice & optogenetic & M & adult & this study \\
\hline & + & two-choice & odor & $\mathrm{F}$ & adult & (Semmelhack and Wang, 2009) \\
\hline \multirow[t]{6}{*}{ Or92a } & + & two-choice & optogenetic & M & adult & (Bell and Wilson, 2016) \\
\hline & 0 & oviposition & olfactogenetics & $\mathrm{F}$ & adult & (Chin et al., 2018) \\
\hline & 0 & two-choice & optogenetic & M & adult & this study \\
\hline & - & two-choice & odor & NA & adult & (Suh et al., 2004) \\
\hline & - & two-choice & odor & $M / F$ & both & (Faucher et al., 2006) \\
\hline & - & two-choice & optogenetic & NA & adult & (Suh et al., 2007) \\
\hline Gr21a & - & two-choice & odor & $\mathrm{F}$ & adult & (Poon et al., 2010) \\
\hline \multirow[t]{5}{*}{ /Gr63a } & - & two-choice & optogenetic & M & adult & (Bell and Wilson, 2016) \\
\hline & 0 & oviposition & olfactogenetics & $\mathrm{F}$ & adult & (Chin et al., 2018) \\
\hline & 0 & two-choice & olfactogenetics & $\mathrm{F}$ & adult & (Chin et al., 2018) \\
\hline & - & two-choice & optogenetic & M & adult & this study \\
\hline & 0 & two-choice & optogenetic & NA & adult & (Suh et al., 2007) \\
\hline \multirow[t]{2}{*}{ Orco } & + & two-choice & optogenetic & M & adult & (Bell and Wilson, 2016) \\
\hline & + & two-choice & optogenetic & M & adult & this study \\
\hline
\end{tabular}


Table S2. Ten-fold cross-validation of ORN-preference prediction models.

RMSE scores were calculated with cross-validation for four models of ORN activity and odor preference. All RMSE scores are close to the standard deviation of the dependent variable $(\sigma=0.23)$.

\begin{tabular}{ll}
\multicolumn{1}{c}{ Model } & RMSE \\
\hline Multiple Linear Regression & 0.22 \\
\hline SVM - Linear & 0.21 \\
\hline SVM - Polynomial & 0.20 \\
\hline SVM - RBF & 0.19 \\
\hline
\end{tabular}

Table S3. Ten-fold cross-validation of single-ORN-valence-weighted prediction models.

Model RMSE

\begin{tabular}{ll}
\hline Multiple Linear Regression & 0.22 \\
\hline SVM - Linear & 0.22 \\
\hline SVM - Polynomial & 0.20 \\
\hline SVM - RBF & 0.20 \\
\hline
\end{tabular}


Table S4. A comparison of the experimental designs of two optogenetic studies investigating ORN-valence behaviour.

\begin{tabular}{lrrr} 
& Bell \& Wilson, 2016 & This study \\
\hline Number of ORNs tested & 8 & 45
\end{tabular}

Total study N of Gal4 controls

0

Total study $\mathrm{N}$ of UAS controls

88

Total study $\mathbf{N}$ of test flies

$\sim 2512$

$\sim 5148$

Independent genetic controls in experiments

No

Yes

Uses valence effect size

No

Yes

Number of stimulus conditions

8

6

Technical replicates per experiment

16

1

Analysis uses technical replicates

Yes

No

Optogenetic light duration (min)

64

9

Opsin type

Channelrhodopsin-2

CsChrimson

Optogenetic light color

Blue

Red

Blind flies

Yes

No

Heat compensation

Yes

No

Highest optogenetic light intensity $\left(\mu \mathrm{W} / \mathrm{mm}^{2}\right)$

1500

72

Optogenetic light-induced heat $\left({ }^{\circ} \mathrm{C}\right)$

Not reported

0.3 
Table S5. A list of ORN-Gal4 lines used in the study.

Gal4 line

\begin{tabular}{|c|c|c|}
\hline Or7a & $w\left[^{*}\right] ; P\{w[+m C]=O r 7 a-G A L 4 . C\} 214 t 1.1$ & 23908 \\
\hline Or9a & $w\left[{ }^{*}\right] ; P\{w[+m C]=O r 9 a-G A L 4 . C\} 106 t 6.1 / T M 3, S b[1]$ & 23918 \\
\hline Or10a & $w\left[{ }^{*}\right] ; P\{w[+m C]=O r 10 a-G A L 4 . F\} 34.2 A ; T M 2 / T M 6 B, T b[1]$ & 9944 \\
\hline Or13a & $w\left[{ }^{*}\right] ; P\{w[+m C]=O r 13 a-G A L 4 . C\} 229 t 56.2 / T M 3, S b[1]$ & 23886 \\
\hline Or19a & $w\left[{ }^{*}\right] ; P\{w[+m C]=O r 19 a-G A L 4 . F\} 61.2$ & 9948 \\
\hline Or19b & $w\left[{ }^{*}\right] ; P\{w[+m C]=O r 19 b-G A L 4 . C\} 218 t 6.1$ & 23889 \\
\hline Or22a & $w\left[{ }^{*}\right] ; P\{w[+m C]=O r 22 a-G A L 4.7 .717\} 14.2$ & 9951 \\
\hline Or22b & $w[1118] ; P\{w[+m C]=O r 22 b-G A L 4.10287\} 105.2$ & 23289 \\
\hline Or23a & $w\left[^{*}\right] ; P\{w[+m C]=O r 23 a-G A L 4.7 .818\} 17.2$ & 9955 \\
\hline$\overline{\text { Or33a }}$ & $w\left[{ }^{*}\right] ; B I[1] / C y O ; P\{w[+m C]=O r 33 a-G A L 4 . F\} 126.4 A$ & 9962 \\
\hline Or33b & $w\left[{ }^{*}\right] ; B I[1] / C y O ; P\{w[+m C]=O r 33 b-G A L 4 . F\} 83.14 / T M 6 B, T b[1]$ & 9963 \\
\hline Or33c & $w\left[{ }^{*}\right] ; P\{w[+m C]=0 r 33 c-G A L 4 . F\} 78.3$ & 9966 \\
\hline Or35a & $w\left[{ }^{*}\right] ; P\{w[+m C]=$ Or35a-GAL4.F $\} 109.3$ & 9968 \\
\hline$\overline{O r 42 a}$ & $w\left[{ }^{*}\right] ; P\{w[+m C]=0 r 42 a-G A L 4 . F\} 48.1$ & 9969 \\
\hline Or42b & $w\left[{ }^{*}\right] ; P\{w[+m C]=O r 42 b-G A L 4 . F\} 64.1$ & 9972 \\
\hline Or43a & $w\left[{ }^{*}\right] ; P\{w[+m C]=0 r 43 a-G A L 4 . W\} 27.6$ & 9974 \\
\hline Or43b & $w\left[^{*}\right] ; P\{w[+m C]=0 r 43 b-G A L 4 . C\} 110 t 6.3$ & 23895 \\
\hline Or46a & $w[*] ; P\{w[+m C]=$ Or46a-GAL4.1.875\}9.10A & 9979 \\
\hline Or47a & $w[*] ; P\{w[+m C]=$ Or47a-GAL4.8.239\} $15.4 A$ & 9982 \\
\hline Or47b & $w\left[{ }^{*}\right] ; P\{w[+m C]=O r 47 b-G A L 4.7 .467\} 15.6$ & 9984 \\
\hline Or49a & $w\left[{ }^{*}\right] ; P\{w[+m C]=0 r 49 a-G A L 4 . F\} 47.2 A$ & 9985 \\
\hline Or49b & $w\left[{ }^{*}\right] ; P\{w[+m C]=0 r 49 b-G A L 4 . F\} 80.1$ & 9986 \\
\hline Or56a & $w\left[^{*}\right] ; P\{w[+m C]=$ Or56a-GAL4.C $\} 113 t 53.2$ & 23896 \\
\hline Or59b & $w\left[{ }^{*}\right] ; P\{w[+m C]=O r 59 b-G A L 4 . C\} 114 t 2.2$ & 23897 \\
\hline$\overline{\text { Or59c }}$ & $w\left[{ }^{*}\right] ; P\{w[+m C]=O r 59 c-G A L 4 . C\} 129 t 1.1$ & 23899 \\
\hline
\end{tabular}




\begin{tabular}{|c|c|c|}
\hline Or65a & $w\left[{ }^{*}\right] ; P\{w[+m C]=O r 65 a-G A L 4 . F\} 72.5$ & 9993 \\
\hline Or65b & $w\left[{ }^{*}\right] ; P\{w[+m C]=O r 65 b-G A L 4 . C\} 198 t 57.1$ & 23902 \\
\hline Or65c & $w\left[{ }^{*}\right] ; P\{w[+m C]=O r 65 c-G A L 4 . C\} 221 t 53.1 / T M 3, S b[1]$ & 23903 \\
\hline Or67a & $w\left[{ }^{*}\right] ; P\{w[+m C]=$ Or67a-GAL4.C $\} 137 t 3.3$ & 23904 \\
\hline Or67b & $w\left[^{*}\right] ; P\{w[+m C]=O r 67 b-G A L 4 . F\} 68.3 / T M 6 B, T b[1]$ & 9995 \\
\hline Or67c & $w\left[{ }^{*}\right] ; P\{w[+m C]=O r 67 c-G A L 4 . C\} 116 t 3.2 / C y O$ & 23905 \\
\hline Or67d & $w\left[{ }^{*}\right] ; P\{w[+m C]=O r 67 d-G A L 4 . F\} 57.2$ & 9998 \\
\hline Or69a & $w\left[{ }^{*}\right] ; P\{w[+m C]=$ Or69a-GAL4.F $\} 81.4$ & 10000 \\
\hline Or71a & $w\left[{ }^{*}\right] ; P\{w[+m C]=O r 71 a-G A L 4 . F\} 30.4$ & 23122 \\
\hline Or82a & $w\left[^{*}\right] ; P\{w[+m C]=O r 82 a-G A L 4 . F\} 135.1 ; T M 2 / T M 6 B, T b[1]$ & 23125 \\
\hline Or83c & $w\left[{ }^{*}\right] ; P\{w[+m C]=O r 83 c-G A L 4 . F\} 73.4 A$ & 23132 \\
\hline Or85a & $w\left[{ }^{*}\right] ; P\{w[+m C]=0 r 85 a-G A L 4 . F\} 67.4$ & 24461 \\
\hline Or85b & $w\left[{ }^{*}\right] ; P\{w[+m C]=O r 85 b-G A L 4 . C\} 179 t 55.1$ & 23912 \\
\hline Or85d & $w[*] ; P\{w[+m C]=O r 85 d-G A L 4 . C\} 143 t 2.1$ & 24148 \\
\hline Or85e & $w[1118] ; P\{w[+m C]=O r 85 e-G A L 4 . W\} 2.19 .1$ & 23293 \\
\hline$\overline{\text { Or85f }}$ & $w\left[{ }^{*}\right] ; P\{w[+m C]=O r 85 f-G A L 4 . F\} 44.6$ & 23136 \\
\hline Or88a & $w\left[{ }^{*}\right] ; P\{w[+m C]=O r 88 a-G A L 4 . F\} 52.1$ & 23137 \\
\hline Or92a & $w\left[{ }^{*}\right] ; P\{w[+m C]=O r 92 a-G A L 4 . F\} 62.1$ & 23139 \\
\hline Or98a & $w\left[{ }^{*}\right] ; P\{w[+m C]=$ Or98a-GAL4.F $\} 115.1$ & 23141 \\
\hline Orco & $w\left[^{*}\right] ; P\{w[+m C]=O r c o-G A L 4 . W\} 11.17 ; T M 2 / T M 6 B, T b[1]$ & 26818 \\
\hline
\end{tabular}




\section{References}

Ache, B.W., and Young, J.M. (2005). Olfaction: diverse species, conserved principles. Neuron 48, 417-430.

Aso, Y., Sitaraman, D., Ichinose, T., Kaun, K.R., Vogt, K., Belliart-Guérin, G., Plaçais, P.-Y., Robie, A.A., Yamagata, N., Schnaitmann, C., et al. (2014). Mushroom body output neurons encode valence and guide memory-based action selection in Drosophila. Elife 3, e04580.

Badel, L., Ohta, K., Tsuchimoto, Y., and Kazama, H. (2016). Decoding of Context-Dependent Olfactory Behavior in Drosophila. Neuron 91, 155-167.

Bell, G. (2016). Replicates and repeats. BMC Biol. 14, 28.

Bell, J.S., and Wilson, R.I. (2016). Behavior Reveals Selective Summation and Max Pooling among Olfactory Processing Channels. Neuron 91, 425-438.

Bellmann, D., Richardt, A., Freyberger, R., Nuwal, N., Schwärzel, M., Fiala, A., and Störtkuhl, K.F. (2010). Optogenetically Induced Olfactory Stimulation in Drosophila Larvae Reveals the Neuronal Basis of Odor-Aversion behavior. Front. Behav. Neurosci. 4, 27.

Bland, J.M., and Altman, D.G. (1999). Measuring agreement in method comparison studies. Stat. Methods Med. Res. 8, 135-160.

Borenstein, M., Hedges, L.V., Higgins, J.P.T., and Rothstein, H.R. (2009). Introduction to Meta-Analysis (Wiley).

Chakraborty, T.S. (2010). Neural correlates of olfactory learning in Drosophila melanogaster.

Chin, S.G., Maguire, S.E., Huoviala, P., Jefferis, G.S.X.E., and Potter, C.J. (2018). Olfactory Neurons and Brain Centers Directing Oviposition Decisions in Drosophila. Cell Rep. 24, 1667-1678.

Collie, A., Maruff, P., Darby, D.G., and MCSTEPHEN, M. (2003). The effects of practice on the cognitive test performance of neurologically normal individuals assessed at brief test-retest intervals. J. Int. Neuropsychol. Soc. 9, 419-428.

Couto, A., Alenius, M., and Dickson, B.J. (2005). Molecular, anatomical, and functional organization of the Drosophila olfactory system. Curr. Biol. 15, 1535-1547.

Cumming, G., and Calin-Jageman, R. (2016). Introduction to the New Statistics: Estimation, Open Science, and Beyond (Routledge).

Davis, R.L. (2007). The scent of Drosophila sex. Neuron 54, 14-16.

Dobritsa, A.A., van der Goes van Naters, W., Warr, C.G., Steinbrecht, R.A., and Carlson, J.R. (2003). Integrating the molecular and cellular basis of odor coding in the Drosophila antenna. Neuron 37, 827-841.

Duchamp-Viret, P., Duchamp, A., and Chaput, M.A. (2003). Single olfactory sensory neurons simultaneously integrate the components of an odour mixture. Eur. J. Neurosci. 18, 2690-2696. 
Dweck, H.K.M., Ebrahim, S.A.M., Kromann, S., Bown, D., Hillbur, Y., Sachse, S., Hansson, B.S., and Stensmyr, M.C. (2013). Olfactory preference for egg laying on citrus substrates in Drosophila. Curr. Biol. 23, 2472-2480.

Ebrahim, S.A.M., Dweck, H.K.M., Stökl, J., Hofferberth, J.E., Trona, F., Weniger, K., Rybak, J., Seki, Y., Stensmyr, M.C., Sachse, S., et al. (2015). Drosophila Avoids Parasitoids by Sensing Their Semiochemicals via a Dedicated Olfactory Circuit. PLoS Biol. 13, e1002318.

Efron, B., Tibshirani, R., Storey, J.D., and Tusher, V. (2001). Empirical Bayes Analysis of a Microarray Experiment. J. Am. Stat. Assoc. 96, 1151-1160.

Eisthen, H.L. (2002). Why are olfactory systems of different animals so similar? Brain Behav. Evol. 59, 273-293.

Evans, C.G. (2019). Scikits-Bootstrap, v1.0.1.

Faucher, C., Forstreuter, M., Hilker, M., and de Bruyne, M. (2006). Behavioral responses of Drosophila to biogenic levels of carbon dioxide depend on life-stage, sex and olfactory context. J. Exp. Biol. 209, 2739-2748.

Fishilevich, E., and Vosshall, L.B. (2005). Genetic and Functional Subdivision of the Drosophila Antennal Lobe.

Gao, Q., Yuan, B., and Chess, A. (2000). Convergent projections of Drosophila olfactory neurons to specific glomeruli in the antennal lobe. Nat. Neurosci. 3, 780-785.

Gao, X.J., Clandinin, T.R., and Luo, L. (2015). Extremely sparse olfactory inputs are sufficient to mediate innate aversion in Drosophila. PLoS One 10, e0125986.

Gelperin, A. (1971). Regulation of Feeding. Annu. Rev. Entomol. 16, 365-378.

van der Goes van Naters, W., and Carlson, J.R. (2007). Receptors and neurons for fly odors in Drosophila. Curr. Biol. 17, 606-612.

Gomez-Diaz, C., Martin, F., Garcia-Fernandez, J.M., and Alcorta, E. (2018). The Two Main Olfactory Receptor Families in Drosophila, ORs and IRs: A Comparative Approach. Front. Cell. Neurosci. 12, 253.

Goodfellow, I., Bengio, Y., and Courville, A. (2016). Deep Learning (MIT Press).

Grabe, V., and Sachse, S. (2018). Fundamental principles of the olfactory code. Biosystems. 164, 94-101.

Groschner, L.N., and Miesenböck, G. (2019). Mechanisms of Sensory Discrimination: Insights from Drosophila Olfaction. Annu. Rev. Biophys. 48, 209-229.

Haddad, R., Weiss, T., Khan, R., Nadler, B., Mandairon, N., Bensafi, M., Schneidman, E., and Sobel, N. (2010). Global features of neural activity in the olfactory system form a parallel code that predicts olfactory behavior and perception. J. Neurosci. 30, 9017-9026.

Hallem, E.A., and Carlson, J.R. (2006). Coding of odors by a receptor repertoire. Cell 125, 143-160.

Haverkamp, A., Hansson, B.S., and Knaden, M. (2018). Combinatorial Codes and Labeled 
Lines: How Insects Use Olfactory Cues to Find and Judge Food, Mates, and Oviposition Sites in Complex Environments. Front. Physiol. 9, 49.

Hernandez-Nunez, L., Belina, J., Klein, M., Si, G., Claus, L., Carlson, J.R., and Samuel, A.D. (2015). Reverse-correlation analysis of navigation dynamics in Drosophila larva using optogenetics. Elife 4.

Hildebrand, J.G., and Shepherd, G.M. (1997). Mechanisms of olfactory discrimination: converging evidence for common principles across phyla. Annu. Rev. Neurosci. 20, 595-631.

Ho, J., Tumkaya, T., Aryal, S., Choi, H., and Claridge-Chang, A. (2019). Moving beyond P values: data analysis with estimation graphics. Nat. Methods.

Howitt, D., and Cramer, D. (2007). Introduction to research methods in psychology (Pearson Education).

Huang, J., Zhang, W., Qiao, W., Hu, A., and Wang, Z. (2010). Functional connectivity and selective odor responses of excitatory local interneurons in Drosophila antennal lobe. Neuron 67, 1021-1033.

Hunter, J.D. (2007). Matplotlib: A 2D Graphics Environment. Comput. Sci. Eng. 9, 90-95.

Jones, E., Oliphant, T., Peterson, P., and Others (2001). SciPy: Open source scientific tools for Python.

Jones, W.D., Cayirlioglu, P., Kadow, I.G., and Vosshall, L.B. (2007). Two chemosensory receptors together mediate carbon dioxide detection in Drosophila. Nature 445, 86-90.

Jung, S.-H., Hueston, C., and Bhandawat, V. (2015). Odor-identity dependent motor programs underlie behavioral responses to odors. Elife 4.

Klapoetke, N.C., Murata, Y., Kim, S.S., Pulver, S.R., Birdsey-Benson, A., Cho, Y.K., Morimoto, T.K., Chuong, A.S., Carpenter, E.J., Tian, Z., et al. (2014). Independent optical excitation of distinct neural populations. Nat. Methods 11, 338-346.

Knaden, M., Strutz, A., Ahsan, J., Sachse, S., and Hansson, B.S. (2012). Spatial representation of odorant valence in an insect brain. Cell Rep. 1, 392-399.

Ko, K.I., Root, C.M., Lindsay, S.A., Zaninovich, O.A., Shepherd, A.K., Wasserman, S.A., Kim, S.M., and Wang, J.W. (2015). Starvation promotes concerted modulation of appetitive olfactory behavior via parallel neuromodulatory circuits. Elife 4.

Koh, H.W.L., Swa, H.L.F., Fermin, D., Ler, S.G., Gunaratne, J., and Choi, H. (2015). EBprot: Statistical analysis of labeling-based quantitative proteomics data. Proteomics 15, 2580-2591.

Kreher, S.A., Mathew, D., Kim, J., and Carlson, J.R. (2008). Translation of sensory input into behavioral output via an olfactory system. Neuron 59, 110-124.

Kuebler, L.S., Schubert, M., Kárpáti, Z., Hansson, B.S., and Olsson, S.B. (2012). Antennal lobe processing correlates to moth olfactory behavior. J. Neurosci. 32, 5772-5782.

Kundu, S., Ganguly, A., Chakraborty, T.S., Kumar, A., and Siddiqi, O. (2016). Synergism and 
Combinatorial Coding for Binary Odor Mixture Perception in Drosophila. eNeuro 3.

Kurtovic, A., Widmer, A., and Dickson, B.J. (2007). A single class of olfactory neurons mediates behavioural responses to a Drosophila sex pheromone. Nature 446, 542-546.

Lai, S.-L., Awasaki, T., Ito, K., and Lee, T. (2008). Clonal analysis of Drosophila antennal lobe neurons: diverse neuronal architectures in the lateral neuroblast lineage. Development 135, 2883-2893.

Larsson, M.C., Domingos, A.I., Jones, W.D., Chiappe, M.E., Amrein, H., and Vosshall, L.B. (2004). Or83b encodes a broadly expressed odorant receptor essential for Drosophila olfaction. Neuron 43, 703-714.

LeDue, E.E., Mann, K., Koch, E., Chu, B., Dakin, R., and Gordon, M.D. (2016).

Starvation-Induced Depotentiation of Bitter Taste in Drosophila. Curr. Biol. 26, 2854-2861.

Lin, H.-H., Cao, D.-S., Sethi, S., Zeng, Z., Chin, J.S.R., Chakraborty, T.S., Shepherd, A.K., Nguyen, C.A., Yew, J.Y., Su, C.-Y., et al. (2016). Hormonal Modulation of Pheromone Detection Enhances Male Courtship Success. Neuron 90, 1272-1285.

Mathew, D., Martelli, C., Kelley-Swift, E., Brusalis, C., Gershow, M., Samuel, A.D.T., Emonet, T., and Carlson, J.R. (2013). Functional diversity among sensory receptors in a Drosophila olfactory circuit. Proc. Natl. Acad. Sci. U. S. A. 110, E2134-E2143.

McCall, R.B., and Appelbaum, M.I. (1973). Bias in the Analysis of Repeated-Measures Designs: Some Alternative Approaches. Child Dev. 44, 401-415.

McKinney, W. (2010). Data structures for statistical computing in python. In Proceedings of the 9th Python in Science Conference, (Austin, TX), pp. 51-56.

Meyer, A., and Galizia, C.G. (2012). Elemental and configural olfactory coding by antennal lobe neurons of the honeybee (Apis mellifera). J. Comp. Physiol. A Neuroethol. Sens. Neural Behav. Physiol. 198, 159-171.

Mohamed, A.A.M., Retzke, T., Das Chakraborty, S., Fabian, B., Hansson, B.S., Knaden, M., and Sachse, S. (2019). Odor mixtures of opposing valence unveil inter-glomerular crosstalk in the Drosophila antennal lobe. Nat. Commun. 10, 1201.

Moon, S.J., Köttgen, M., Jiao, Y., Xu, H., and Montell, C. (2006). A taste receptor required for the caffeine response in vivo. Curr. Biol. 16, 1812-1817.

Parnas, M., Lin, A.C., Huetteroth, W., and Miesenböck, G. (2013). Odor discrimination in Drosophila: from neural population codes to behavior. Neuron 79, 932-944.

Pedregosa, F., Varoquaux, G., Gramfort, A., Michel, V., Thirion, B., Grisel, O., Blondel, M., Prettenhofer, P., Weiss, R., Dubourg, V., et al. (2011). Scikit-learn: Machine learning in Python. The Journal of Machine Learning Research 12, 2825-2830.

Poon, P.C., Kuo, T.-H., Linford, N.J., Roman, G., and Pletcher, S.D. (2010). Carbon dioxide sensing modulates lifespan and physiology in Drosophila. PLoS Biol. 8, e1000356.

Riffell, J.A., Lei, H., Christensen, T.A., and Hildebrand, J.G. (2009). Characterization and 
coding of behaviorally significant odor mixtures. Curr. Biol. 19, 335-340.

Ronderos, D.S., Lin, C.-C., Potter, C.J., and Smith, D.P. (2014). Farnesol-detecting olfactory neurons in Drosophila. J. Neurosci. 34, 3959-3968.

Root, C.M., Ko, K.I., Jafari, A., and Wang, J.W. (2011). Presynaptic facilitation by neuropeptide signaling mediates odor-driven food search. Cell 145, 133-144.

Semmelhack, J.L., and Wang, J.W. (2009). Select Drosophila glomeruli mediate innate olfactory attraction and aversion. Nature 459, 218-223.

Sengupta, P. (2013). The belly rules the nose: feeding state-dependent modulation of peripheral chemosensory responses. Curr. Opin. Neurobiol. 23, 68-75.

Shang, Y., Claridge-Chang, A., Sjulson, L., Pypaert, M., and Miesenböck, G. (2007). Excitatory local circuits and their implications for olfactory processing in the fly antennal lobe. Cell $128,601-612$.

Shao, L., Saver, M., Chung, P., Ren, Q., Lee, T., Kent, C.F., and Heberlein, U. (2017). Dissection of the Drosophila neuropeptide $\mathrm{F}$ circuit using a high-throughput two-choice assay. Proc. Natl. Acad. Sci. U. S. A. 114, E8091-E8099.

Shen, K., Tootoonian, S., and Laurent, G. (2013). Encoding of mixtures in a simple olfactory system. Neuron $80,1246-1262$.

Silbering, A.F., and Galizia, C.G. (2007). Processing of odor mixtures in the Drosophila antennal lobe reveals both global inhibition and glomerulus-specific interactions. J. Neurosci. 27, 11966-11977.

Stensmyr, M.C., Dweck, H.K.M., Farhan, A., Ibba, I., Strutz, A., Mukunda, L., Linz, J., Grabe, V., Steck, K., Lavista-Llanos, S., et al. (2012). A conserved dedicated olfactory circuit for detecting harmful microbes in Drosophila. Cell 151, 1345-1357.

Suh, G.S.B., Wong, A.M., Hergarden, A.C., Wang, J.W., Simon, A.F., Benzer, S., Axel, R., and Anderson, D.J. (2004). A single population of olfactory sensory neurons mediates an innate avoidance behaviour in Drosophila. Nature 431, 854-859.

Suh, G.S.B., Ben-Tabou de Leon, S., Tanimoto, H., Fiala, A., Benzer, S., Anderson, D.J., Wang, Y., Chiang, A.S., Xia, S., Kitamoto, T., et al. (2007). Light Activation of an Innate Olfactory Avoidance Response in Drosophila. Curr. Biol. 17, 905-908.

Temasek Life Sciences Laboratories (2018). Recipe for Drosophila media.

Thoma, M., Hansson, B.S., and Knaden, M. (2014). Compound valence is conserved in binary odor mixtures in Drosophila melanogaster. J. Exp. Biol. 217, 3645-3655.

Tumkaya, T., Ott, S., and Claridge-Chang, A. (2018). Systematic review of Drosophila short-term-memory genetics: Meta-analysis reveals robust reproducibility. Neurosci. Biobehav. Rev.

Tumkaya, T., Stewart, J., Burhanudin, S., and Claridge-Chang, A. (2019). Optogenetic olfactory behavior depends on illumination characteristics. 
Turner, S.L., and Ray, A. (2009). Modification of CO2 avoidance behaviour in Drosophila by inhibitory odorants. Nature 461, 277-281.

Vosshall, L.B., Wong, A.M., and Axel, R. (2000). An olfactory sensory map in the fly brain. Cell 102, 147-159.

Wang, J.W., Wong, A.M., Flores, J., Vosshall, L.B., and Axel, R. (2003). Two-photon calcium imaging reveals an odor-evoked map of activity in the fly brain. Cell 112, 271-282.

Wang, K., Gong, J., Wang, Q., Li, H., Cheng, Q., Liu, Y., Zeng, S., and Wang, Z. (2014). Parallel pathways convey olfactory information with opposite polarities in Drosophila. Proc. Natl. Acad. Sci. U. S. A. 111, 3164-3169.

Waskom, M., Botvinnik, O., O’Kane, D., Hobson, P., Lukauskas, S., Gemperline, D.C., Augspurger, T., Halchenko, Y., Cole, J.B., Warmenhoven, J., et al. (2017). mwaskom/seaborn: v0.8.1 (September 2017).

Wilson, R.I. (2008). Neural and behavioral mechanisms of olfactory perception. Curr. Opin. Neurobiol. 18, 408-412.

Wong, A.M., Wang, J.W., and Axel, R. (2002). Spatial representation of the glomerular map in the Drosophila protocerebrum. Cell 109, 229-241.

Zhou, S., Mackay, T.F.C., and Anholt, R.R.H. (2010). Tuning the chemosensory window: a fly's perspective. Fly 4, 230-235. 